\title{
Inactivation of paracellular cation-selective claudin-2 channels attenuates immune-mediated experimental colitis in mice
}

\author{
Preeti Raju, ${ }^{1}$ Nitesh Shashikanth, ${ }^{1}$ Pei-Yun Tsai, ${ }^{2}$ Pawin Pongkorpsakol, ${ }^{1}$ Sandra Chanez-Paredes, ${ }^{1}$ Peter R. Steinhagen, ${ }^{1}$ \\ Wei-Ting Kuo, ${ }^{1}$ Gurminder Singh, ${ }^{1,2}$ Sachiko Tsukita, ${ }^{3}$ and Jerrold R. Turner ${ }^{1,2}$ \\ 'Laboratory of Mucosal Barrier Pathobiology, Department of Pathology, Brigham and Women's Hospital and Harvard Medical School, Boston, Massachusetts, USA. ²Department of Pathology, University of \\ Chicago, Chicago, Illinois, USA. 'Laboratory of Biological Science, Graduate School of Frontier Biosciences and Graduate School of Medicine, Osaka University, Osaka, Japan.
}

\begin{abstract}
The tight junction protein claudin-2 is upregulated in disease. Although many studies have linked intestinal barrier loss to local and systemic disease, these have relied on macromolecular probes. In vitro analyses show, however, that these probes cannot be accommodated by size- and charge-selective claudin-2 channels. We sought to define the impact of claudin-2 channels on disease. Transgenic claudin-2 overexpression or IL-13-induced claudin-2 upregulation increased intestinal small cation permeability in vivo. IL-13 did not, however, affect permeability in claudin-2-knockout mice. Claudin-2 is therefore necessary and sufficient to effect size- and charge-selective permeability increases in vivo. In chronic disease, T cell transfer colitis severity was augmented or diminished in claudin-2-transgenic or -knockout mice, respectively. We translated the in vitro observation that casein kinase-2 (CK2) inhibition blocks claudin-2 channel function to prevent acute, IL-13induced, claudin-2-mediated permeability increases in vivo. In chronic immune-mediated colitis, CK2 inhibition attenuated progression in claudin-2-sufficient, but not claudin-2-knockout, mice, i.e., the effect was claudin-2 dependent. Paracellular flux mediated by claudin-2 channels can therefore promote immune-mediated colitis progression. Although the mechanisms by which claudin- 2 channels intensify disease remain to be defined, these data suggest that claudin-2 may be an accessible target in immune-mediated disorders, including inflammatory bowel disease.
\end{abstract}

\section{Introduction}

Barriers that define distinct tissue compartments and separate self from non-self are necessary for survival of multicellular organisms. In some organs, for example the integument and bladder, the barrier is nearly impermeable to water, ions, and macromolecules. Other sites, however, including the intestine and renal tubules, require selectively permeable barriers (1-4). Both selective permeability and barrier function are defined by the epithelial tight junction $(5,6)$.

Paracellular permeability reflects the sum of separate tight junction pore and leak pathways. The pore pathway is a highcapacity, size- and charge-selective route whose permeability is primarily defined by the subset of claudin family proteins expressed (7-9). In contrast, the low-capacity, non-chargeselective leak pathway is permeable to macromolecules with diameters up to approximately $125 \AA$. Nearly all studies of in vivo intestinal barrier function have relied on large probes, e.g., 4-kDa FITC-dextran (28 A diameter), that can cross the leak

\section{Delated Commentary: p. 5100}

Authorship note: PR and NS contributed equally to this work. Conflict of interest: JRT is a cofounder of, and shareholder in, Thelium Therapeutics. Copyright: ( 2020, American Society for Clinical Investigation. Submitted: April 1, 2020; Accepted: June 3, 2020; Published: August 24, 2020 Reference information: J Clin Invest. 2020;130(10):5197-5208. https://doi.org/10.1172/JCl138697. pathway but are excluded by the pore pathway, which has a maximum diameter of approximately $8 \AA$.

Claudin-2, a prototypic pore-forming claudin, forms actively gated channels that are selective for water and small cations (1014). Despite forming a high-capacity cation and water channel in vitro $(11,12,15,16)$, claudin-2 function has been difficult to define in vivo. Although claudin-2 is expressed in epithelial cells lining the small intestine, colon, pancreatobiliary tree, renal tubules, and, possibly, seminiferous tubules (17-20), claudin-2 knockout induces subtle phenotypic changes that are only apparent under stress $(3,21,22)$. This may, in part, reflect some functional redundancy between claudin-2 and claudin-15, which also forms paracellular channels that are selective for small cations and water $(10,13,16,22-24)$.

Within the intestinal epithelium, claudin-2 is highly expressed at birth but rapidly downregulated, in concert with claudin-15 upregulation, at the time of weaning in rodents and humans (22, $25,26)$. Intestinal epithelial claudin-2 expression is reactivated in inflammatory states including celiac disease (26-28), infectious enterocolitis (29), and inflammatory bowel disease (IBD), where the degree of upregulation correlates directly with disease severity (30-32). Although previous studies have shown that preservation of the leak pathway barrier to macromolecular flux, e.g., by myosin light chain kinase inhibition, limits both claudin-2 upregulation and immune-mediated colitis severity (33-39), contributions of the pore pathway to disease development have not been defined. Nevertheless, it has been hypothesized that claudin-2 
upregulation contributes to tissue injury and promotes disease progression $(27,30,40)$.

The impact of claudin-2 upregulation in disease has been studied in Citrobacter rodentium infectious colitis (29) and chemical (dextran sulfate sodium, DSS) colitis models $(41,42)$ using claudin-2-transgenic (Cldn2 $\left.2^{T g}\right)$ and -knockout $\left(C l d n 2^{-/-}\right)$mice. (Although Cldn2 is X-linked, for simplicity male and female mice

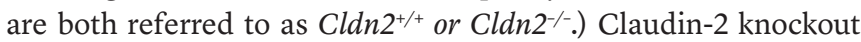
augmented disease severity, while claudin-2 overexpression was protective, in both infectious and chemical colitis models. This contrasts sharply with the effects of increased leak pathway permeability and suggests that claudin-2-mediated pore pathway flux may be an adaptive response that promotes mucosal repair and homeostasis. Claudin-2 function has not, however, been studied in the context of immune-mediated colitis, thereby leaving the question of how claudin-2 upregulation might impact inflammatory diseases unanswered.

Here, we assessed the effects of intestinal epithelial claudin-2 overexpression or knockout on in vivo pore pathway permeability and progression of immune-mediated, experimental IBD. Claudin-2 upregulation was required for cytokine-induced pore pathway permeability increases. Immune-mediated colitis severity was intensified in $\mathrm{Cldn} 2^{T g} \mathrm{Rag1}^{-/-}$mice but attenuated by either genetic claudin-2 deletion or pharmacological claudin-2 channel inactivation. These data illuminate the differences between distinct forms of paracellular flux and indicate that discrimination between pore and leak pathway regulation is critical to advancing our understanding of disease-associated barrier loss. The data also provide insight into the impact of claudin-2 expression in human diseases, including IBD, and the therapeutic potential of claudin-2 channel inhibition.

\section{Results}

Claudin-2 expression is necessary and sufficient for IL-13-induced barrier loss in vivo. We and others have shown that intestinal epithelial claudin-2 expression, which is limited in adults, can be upregulated in response to IL-13 in vitro and in vivo $(26,30,31,43)$. This IL-13-induced claudin-2 expression enhances pore pathway permeability of cultured monolayers in vitro $(26,30,31,43)$. To define the impact of IL-13 and contributions of claudin-2 to paracellular permeability in vivo, we analyzed intestinal tissues from Cldn2 $2^{+/+}$and Cldn2 $2^{-/-}$mice. Neither IL-13 treatment nor claudin-2 knockout affected colonic histopathology (Figure 1A). As expected, IL-13 markedly increased claudin-2 expression within colonic crypt epithelia of $C l d n 2^{+/+}$, but not $C l d n 2^{-/-}$mice (Figure 1B). Quantitative analysis showed that claudin-2 expression in IL-13-treated mice was increased 1.9-fold compared with vehicle-treated mice (Figure 1, C and D). In contrast, expression of other tight junction proteins was unaffected by IL-13 treatment (Figure 1, C and D).

Claudin-2 expression, whether induced genetically or by cytokines, specifically enhances flux across the charge- and sizeselective pore pathway but not the less selective leak pathway in vitro $(14,15,43-46)$. To define the impact of IL-13-induced claudin-2 upregulation in vivo, bi-ionic potentials were measured to determine paracellular permeability of $\mathrm{Na}^{+}$and larger monovalent cations $(14,45,47)$. IL-13 increased paracellular permeability of $\mathrm{Na}^{+}$, methylamine, and, to a lesser extent, ethylamine, but not the larger cations tetramethylammonium, tetraethylammonium, or $N$-methyl-D-glucamine (Figure 1E). This size-selective permeability increase is characteristic of claudin-2 channel activity (14-16, 48-50), but does not exclude the possibility that other cation-selective pore-forming claudins, e.g., claudin-15 (10, 13, 22), contribute to IL-13-induced barrier loss in vivo. We therefore asked if claudin-2 was required for IL-13-induced barrier loss. Prior to IL-13 treatment, paracellular permeability was similar

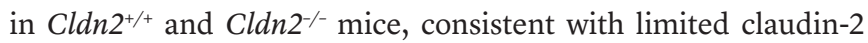
expression in adults. IL-13 treatment was not, however, able to increase paracellular permeability of small cations, including $\mathrm{Na}^{+}$and methylamine in Cldn2 $2^{-/}$mice (Figure 1F). Claudin-2 is therefore necessary for IL-13-induced increases in intestinal pore pathway permeability.

In order to determine if claudin-2 upregulation is sufficient to mediate the effects of IL-13 on intestinal cation permeability, transgenic mice expressing GFP-tagged mouse claudin-2 from the intestinal epithelium-specific villin (Vil1) promoter (Cldn $2^{T g}$ ) were characterized (29). Total claudin-2 expression (the sum of endogenous and GFP-claudin-2) in these $C l d n 2^{T g}$ mice was similar in distribution (Figure $1 \mathrm{G}$ ) to that of IL-13-treated Cldn2 ${ }^{+/+}$mice (Figure 1B) and increased 3.3-fold relative to $\mathrm{Cldn2^{+/+ }}$ mice that did not receive IL-13 (Figure 1, H and I). Moreover, transgenic GFPclaudin-2 expression selectively increased paracellular permeability of $\mathrm{Na}^{+}$, methylamine, and, to a lesser extent, ethylamine, in a manner that recapitulated the effect of IL-13 on Cldn $2^{+/+}$mice (Figure 1J). Claudin-2 is, therefore, both necessary and sufficient to mediate IL-13-induced barrier loss in vivo.

Transgenic claudin-2 expression exacerbates immune-mediated colitis. We have found that severity and duration of infectious colitis are reduced in these GFP-Cldn2 ${ }^{T g}$ mice (29). A study of similar transgenic mice expressing human claudin-2 from the same Vil1 promoter demonstrated that claudin-2 overexpression was also protective in DSS-induced, chemical colitis (41). Conversely, claudin-2 knockout exacerbated infectious and chemical colitis $(29,42)$. These data suggest that claudin-2-mediated pore pathway permeability increases may be adaptive, i.e., beneficial in IBD. To test this hypothesis, we compared severity of experimental IBD in immunodeficient $\mathrm{Cldn} 2^{T g} \mathrm{Rag1}^{-/-}$and $\mathrm{Cldn} 2^{+/+} \mathrm{Rag1}^{-/-}$mice using the well-established $\mathrm{T}$ cell transfer model $(33,51)$.

Unexpectedly, disease was significantly more severe in $C l d n 2^{T g}$ $\operatorname{Rag1}^{1 /-}$ mice relative to $\mathrm{Cldn2^{+/+ }} \mathrm{Ragl}^{-/-}$mice. This was demonstrated by more extensive weight loss (Figure 2A) and greater disease activity (Figure 2B). Consistent with increased disease severity, leak (macromolecular) and unrestricted (epithelial damage) pathway permeabilities, measured as $4-\mathrm{kDa}$ and $70-\mathrm{kDa}$ dextran flux, respectively, were markedly greater in $\mathrm{Cldn} 2^{\mathrm{Tg}} \mathrm{Rag1}^{-/-}$mice relative to $C l d n 2^{+/+} \operatorname{Rag1}^{1^{-/}}$mice (Figure $2 \mathrm{C}$ and ref. 29). Colitis was associated with increased claudin-2 expression in $\mathrm{Cldn2^{+/+ }} \mathrm{Rag1}^{-/-}$and Cldn2 ${ }^{\text {Tg }} \mathrm{Rag1}^{-/-}$mice (Figure 2D). In colitic Cldn2 $2^{+/+} \mathrm{Rag1}^{1^{-/}}$mice, the region of claudin- 2 expression extended beyond crypt bases to include the entire lower half of elongated crypts, i.e., the transit-amplifying zone. The region of endogenous claudin-2 expression was similarly increased in colitic Cldn $2^{\mathrm{Tg}} \mathrm{Rag}^{-/-}$mice, but these mice also expressed transgenic, GFP-claudin-2 in the upper half of the crypt and surface epithelium (Figure 2D). It is possible that this expanded area of claudin-2 expression contrib- 
A

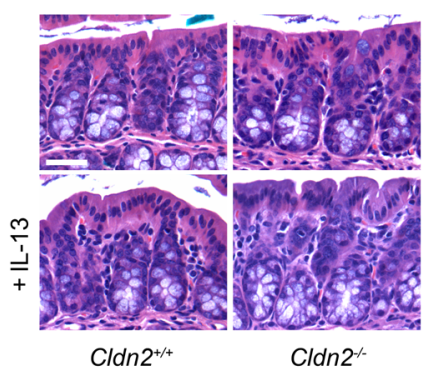

C

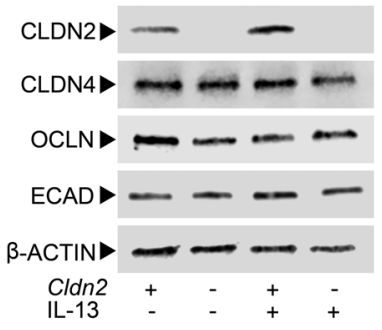

B

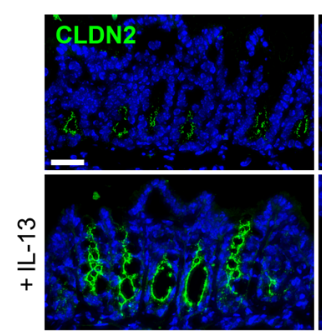

$C / d n 2^{+/+}$

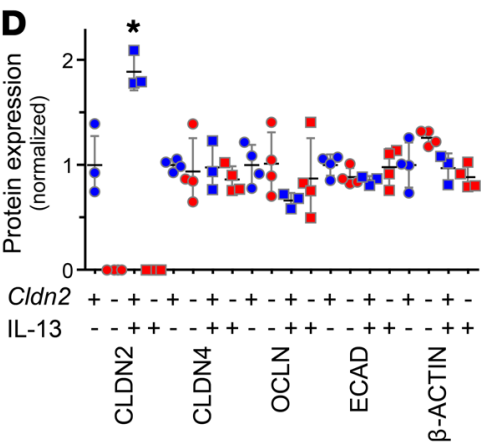

E

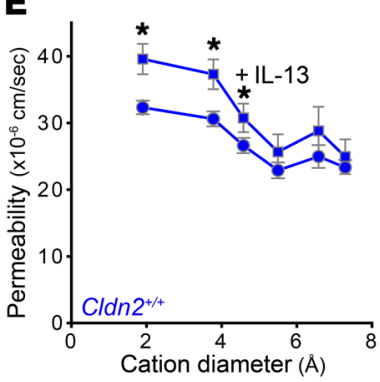

$\mathbf{F}$

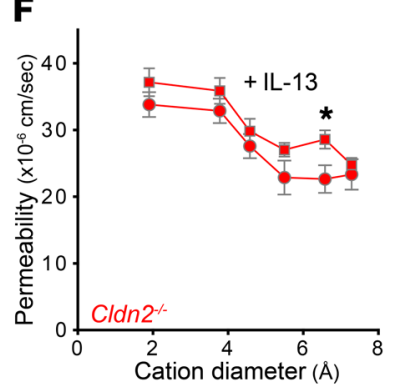

G

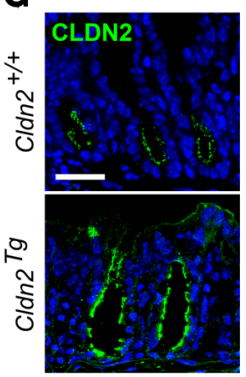

H

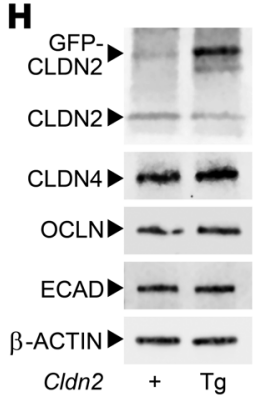

I

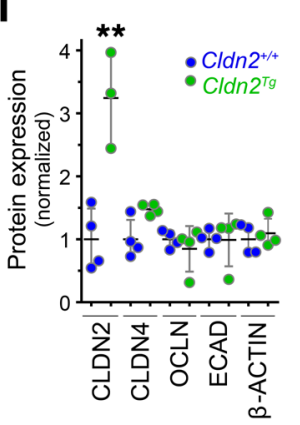

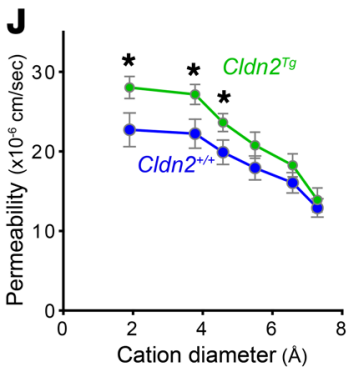

Figure 1. Claudin-2 is necessary and sufficient for IL-13induced changes in pore pathway permeability. (A)

Colonic histopathology of $\mathrm{Cldn2}^{+/+}$or $\mathrm{Cldn2}^{-/-}$mice was not affected by injection with vehicle or IL-13. (B) IL-13 increases claudin-2 (CLDN2, green) protein expression

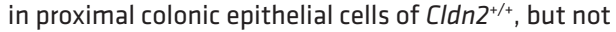
Cldn2 $2^{-/-}$, mice. Nuclei (blue) are shown for reference. (C) Immunoblots of isolated colonic epithelia from Cldn2 ${ }^{+/+}$

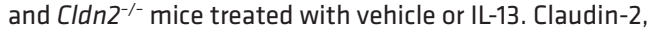
claudin-4 (CLDN4), occludin (OCLN), E-cadherin (ECAD), and $\beta$-actin are shown. (D) Densitometry of immunoblots, as in C. $n=3-4$ per condition. ANOVA with Bonferroni's correction. (E and $\mathbf{F}$ ) Proximal colonic mucosae from Cldn2 $2^{+/+}(\mathbf{E})$ and $\mathrm{Cldn2} 2^{-/-}(\mathbf{F})$ mice treated with vehicle (circles) or IL-13 (squares) were mounted in Ussing chambers for analysis of paracellular permeability. Bi-ionic potential measurements were used to determine the permeabilities of $\mathrm{Na}^{+}$and 5 larger cations (methylamine, ethylamine, tetramethylammonium, tetraethylammonium, and $\mathrm{N}$-methyl-D-glucamine). IL-13 increased permeability of $\mathrm{Na}^{+}$, methylamine, and ethylamine, but not larger cations, in $\mathrm{Cldn2^{+/+ }}$ mice. IL-13 did not affect $\mathrm{Na}^{+}$, methylamine, or ethylamine permeability in Cldn2 $2^{-/-}$mice. $n=8$ and 9 for $\mathrm{Cldn2^{+/+ }}$ mice without or with IL-13 treatment, respectively, and $n=5$

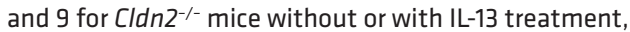
respectively. Data compiled from 3 independent experiments. Two-tailed $t$ test. (G) Claudin-2 (green) expression in $\mathrm{Cldn2^{+/+ }}$ and $\mathrm{Cldn} 2^{T g}$ mice. (H) Representative immunoblots of isolated colonic epithelia from Cldn2 $2^{+/+}$ and $\mathrm{Cldn} 2^{\text {Tg }}$ mice. (I) Densitometry of immunoblots of isolated colonic epithelia from Cldn2 $2^{+/+}$and Cldn $2^{\text {Tg }}$ mice, as in $\mathbf{H} . n=3-4$ per condition. Two-tailed $t$ test. (J) Ussing chamber analysis (as in E) of proximal colonic mucosae from Cldn2 $2^{+/+}$and Cldn2 $2^{\text {Tg }}$ mice. Claudin-2 overexpression selectively increased $\mathrm{Na}^{+}$, methylamine, and

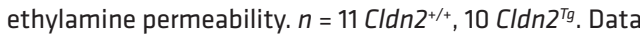
compiled from 3 independent experiments. Two-tailed $t$ test. ${ }^{*} P<0.05$; ${ }^{*} P<0.01$. Scale bars: $50 \mu \mathrm{m}$. uted to earlier increases in fecal water content in $\mathrm{Cldn}^{\mathrm{Tg}} \mathrm{Rag1}^{-/-}$, relative to $\mathrm{Cldn2}^{+/+} \mathrm{Rag1}^{-/-}$, mice (Figure $2 \mathrm{E}$ ).

Consistent with greater weight loss and higher disease activity scores, mucosal IFN- $\gamma$ (Figure 2F) and TNF (Figure 2G), T cell recruitment (Figure $2 \mathrm{H}$ ), and histopathology scores (Figure 2I) were increased in $\mathrm{Cldn}^{\mathrm{Tg}} \mathrm{Rag1}^{-/-}$mice relative to $\mathrm{Cldn2^{+/+ }}$ Rag1 $^{-/-}$mice. Thus, in contrast to infectious and chemical colitis, intestinal epithelial claudin-2 overexpression accelerates onset and enhances severity of experimental IBD, i.e., chronic immunemediated colitis.

Claudin-2 deficiency limits progression of immune-mediated colitis. The increased severity of immune-mediated disease in
Cldn2 ${ }^{T g}$ Rag1 $^{-/-}$mice was unanticipated. We therefore assessed the impact of claudin-2 knockout on immune-mediated experimental colitis. Disease onset, as defined by weight loss (Figure $3 \mathrm{~A})$ and disease activity (Figure 3B), was markedly delayed in Cldn2 $2^{-/}$Rag1/- mice, which were also largely protected from increases in leak pathway permeability (Figure 3C). T cell transfer increased claudin-2 expression in $\mathrm{Cldn}^{+/+} \mathrm{Rag1}^{-/-}$but not Cldn $2^{-/-}$Rag1 $^{-/-}$mice (Figure 3D), and fecal water increases were delayed in the absence of claudin-2 (Figure 3E). Finally, mucosal cytokine production (Figure 3, F and G), T cell recruitment (Figure $3 \mathrm{H}$ ), and histopathology (Figure 3I) were markedly reduced in $\mathrm{Cldn}^{-/-} \mathrm{Rag1}^{-/-}$mice. Genetic claudin-2 inhibition therefore 
A
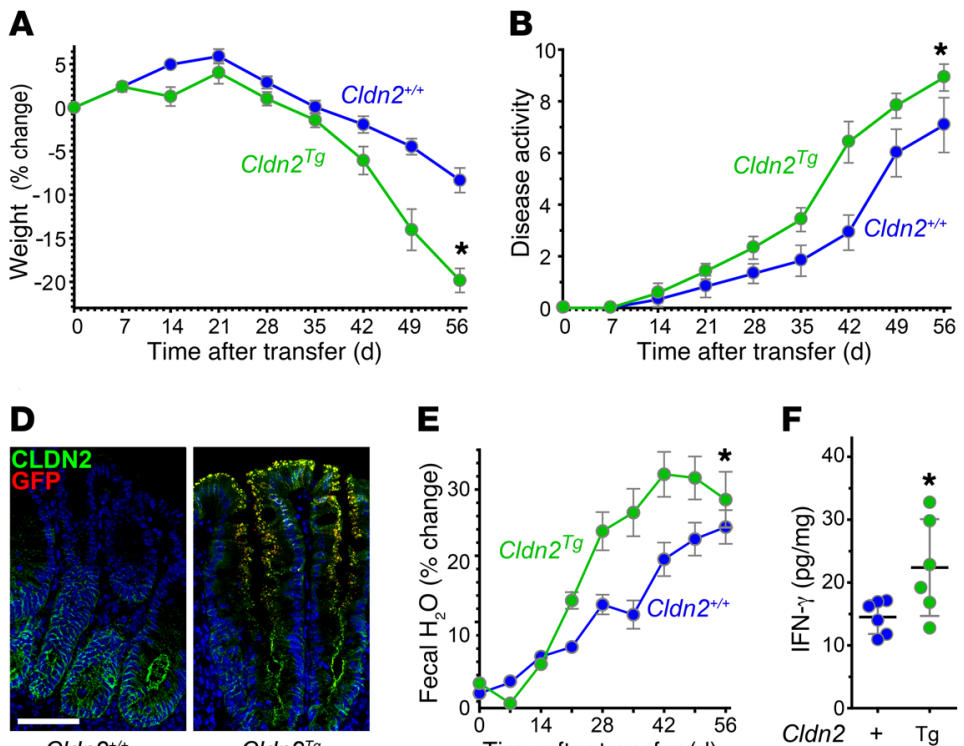

Cldn2 ${ }^{T g}$

H

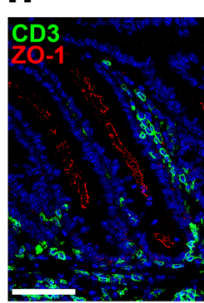

$\mathrm{Cldn2} 2^{+/+}$

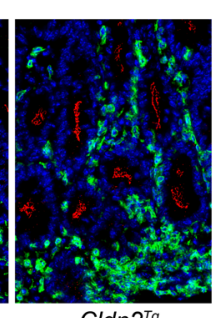

$\mathrm{Cldn2^{ \text {Tg } }}$
E

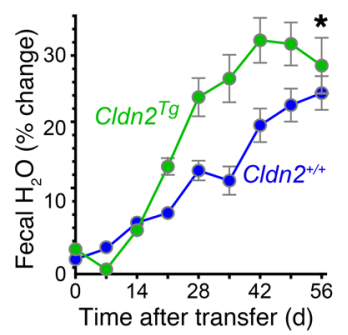

F

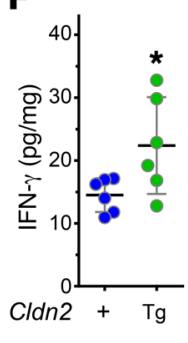

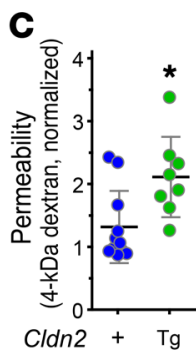

G

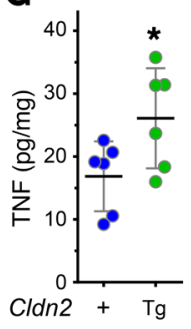

Figure 2. Intestinal epithelium-specific claudin-2 overexpression exacerbates immune-mediated colitis. (A) Weight loss and (B) disease activity following T cell transfer were both greater in $\mathrm{Cldn}^{\mathrm{Tg}_{g}} \mathrm{Rag}^{1 /-}$ mice (Cldn $2^{\text {Tg }}$, green circles) relative to $\mathrm{Cldn2^{+/+ }}$ Rag $^{1 /-}$ mice (Cldn $2^{+/+}$, blue circles). $n=12$ per genotype. Two-tailed $t$ test on day 56. (C) Consistent with greater tissue injury, increases in 4-kDa dextran permeability were amplified in $\mathrm{Cldn}^{\mathrm{Tg}_{9}} \mathrm{Rag}^{\text {-1/- }}$ mice relative to $\mathrm{Cldn2^{+/+ }}$ Rag $1^{-1-}$ mice. $n=12$ per genotype. Two-tailed $t$ test. (D) Claudin-2 expression per cell as well as the number of claudin-2-expressing cells in each crypt increased markedly on day 56. Endogenous claudin-2 (CLDN2, green) was limited to the bottom half of the crypt in both Cldn2 $2^{+/}$Rag $^{-/-}$and Cldn $2^{\text {Tg }}$ Rag $^{-/-}$mice. In contrast, GFP-claudin-2 (red) was detected from the mid crypt to the mucosal surface. (E) Fecal water of Cldn2 $2^{+/+}$Rag1 $^{-/-}$and Cldn2 $2^{T_{g}}$ Rag $1^{-1-}$ mice following T cell transfer. $n=12$ per genotype. Two-tailed $t$ test. (F) Mucosal IFN- $\gamma$ and (G) TNF on day 56. $n=6$ per genotype. Two-tailed $t$ test. (H) Representative immunostain of proximal colon showing CD3 (green) and ZO-1 (red) and corresponding quantitative analysis. $n=10$ per genotype. Two-tailed $t$ test. (I) Representative pathology scores on day 56. $n=7 \mathrm{Cldn}^{+/+} \mathrm{Rag1}^{1 /-}$, $10 \mathrm{Cldn}^{\mathrm{T}_{\mathrm{g}}} \mathrm{Rag}^{1 /-1}$. Two-tailed $t$ test. Data presented in this figure are typical of 3 independent experiments. ${ }^{*} P<0.05 ;{ }^{*} P<0.01$. Scale bars: $50 \mu \mathrm{m}$. reduces disease progression, suggesting that claudin-2 may be a viable therapeutic target.

Despite reduced disease severity, mortality is increased by claudin-2 deficiency. Surprisingly, T cell transfer caused much greater mortality in $\mathrm{Cldn2}^{-/-} \mathrm{Rag1}^{-/-}$mice relative to $\mathrm{Cldn2}^{+/+}$ Rag1 $^{-/-}$mice (Figure 4A). The manner of death was, however, atypical. In contrast to $\mathrm{Cldn2^{+/+ }} \mathrm{Rag1}^{-/-}$mice, which died following progressive disease, $\mathrm{Cldn2^{-/- }} \mathrm{Rag1}^{-/-}$mice were well until approximately 6 weeks after $\mathrm{T}$ cell transfer, at which time a subset became inactive and hunched, despite limited weight loss, and died within days. This distinct pattern of disease before death of $\mathrm{Cldn2}^{-/-} \mathrm{Rag1}^{-/-}$mice was explained upon necropsy. Gross intestinal obstruction (Figure 4B) with ischemic injury superimposed on experimental IBD (Figure 4C) was present in $50 \%$ of $\mathrm{Cldn2}^{-/-} \mathrm{Rag1}^{-/-}$mice that died. In contrast, intestinal obstruction was not present in any $\mathrm{Cldn2^{+/+ }} \mathrm{Rag1}^{-/-}$mice. Thus, even though it reduced colitis severity, claudin-2 knockout promoted obstruction that compromised overall survival.

Fibrostenosing strictures commonly cause intestinal obstruction in Crohn's disease. Picrosirius red staining (Figure 4D) was used to assess fibrosis in colons from Cldn2 $2^{-/-} \operatorname{Rag1}^{-/-}$and Cldn2 $2^{+/+}$Rag1 $^{-/-}$mice; only minimal fibrosis was detected (Figure $4 \mathrm{E})$. Fibrosis cannot, therefore, explain intestinal obstruction in Cldn2 $2^{-/} \mathrm{Rag1}^{-/-}$mice. Dysmotility was also considered as a potential cause of obstruction. No differences in small intestinal (Figure $4 \mathrm{~F}$ ) or colonic (Figure $4 \mathrm{G}$ ) motility were, however, detected between $C l d n 2^{T g}, C l d n 2^{+/+}$, and Cldn2 $2^{--}$mice. The intestinal obstruction observed in $\mathrm{Cldn2^{-/- }} \operatorname{Rag}^{-/-}$mice is not, therefore, secondary to fibrosis or dysmotility.

Insufficient fecal hydration leads to increased mortality of Cldn2 $2^{-/}$Rag1 ${ }^{-/-}$mice. Having excluded motility and structural alterations of the bowel wall, we asked if insufficient luminal hydration could lead to obstruction in Cldn2-/ $\mathrm{Ragl}^{-/-}$mice. Although fecal water increased during experimental IBD progression in $\mathrm{Cldn2}^{-/-} \mathrm{Rag1}^{-/-}$mice, it was markedly attenuated relative to $\mathrm{Cldn2}^{+/+} \mathrm{Rag1}^{-/-}$mice (Figure 3E). In contrast, fecal $\mathrm{Na}^{+}$did not increase during experimental IBD in $\mathrm{Cldn}^{-{ }^{--}} \mathrm{Rag1}^{-/-}$ mice (Figure 5A). To better understand this difference, we assessed expression of the two claudins, 2 and 15, that mediate paracellular $\mathrm{Na}^{+}$and water flux in the intestine as well as ZO-1 and occludin (Figure 5B). Immune-mediated disease induced marked claudin-15 upregulation in $\mathrm{Cldn2}^{-/-} \mathrm{Rag1}^{-/-}$, but not $\mathrm{Cldn}^{+/+} \mathrm{Rag1}^{-/-}$, mice (Figure 5, B and C). Conceptually, claudin-15 upregulation could reflect an effort to compensate for the lack of claudin-2 $(4,22)$. Claudin-15 was not, however, able to complement claudin-2 knockout, as fecal $\mathrm{Na}^{+}$and water did not increase in $\mathrm{Cldn2}^{-/-} \mathrm{Rag1}^{-/-}$mice. This is surprising, as available data indicate that claudin-2 and claudin-15 are, at least in part, functionally redundant in vitro and in vivo $(4,13,26,52)$. Nevertheless, the data indicate that these closely related claudins must have distinct biophysical properties (13) or mechanisms of pore regulation (50) that allow claudin-2, but not claudin-15, to increase fecal $\mathrm{Na}^{+}$and water during progres- 
A

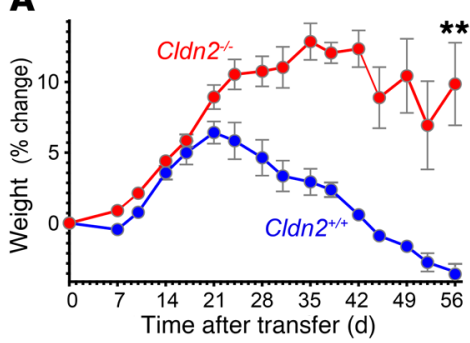

D

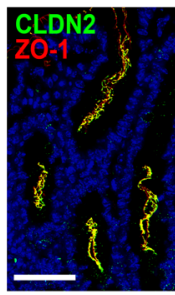

$\mathrm{Cldn2} 2^{+/+}$

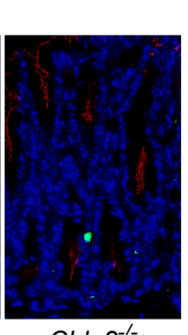

Cldn2 $2^{-/-}$
E

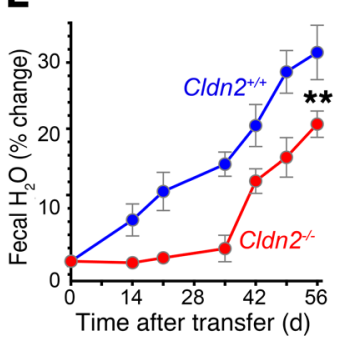

B

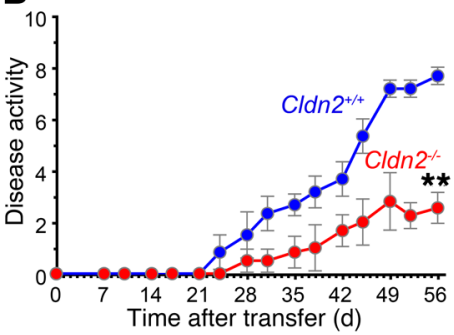

$\mathbf{F}$

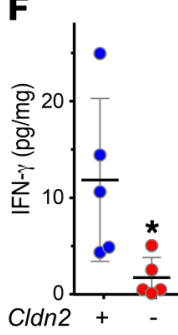

C

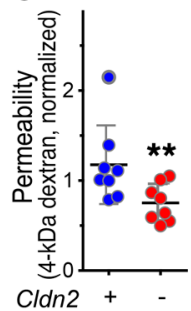

G

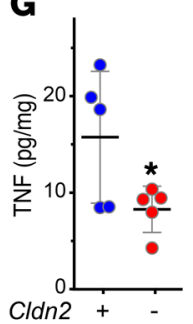

H

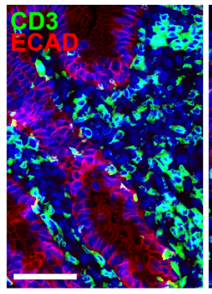

Cldn2 $2^{+/+}$

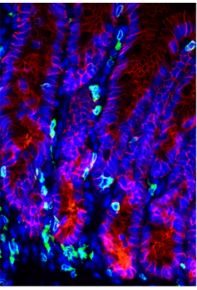

Cldn2-1-

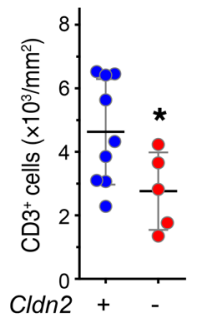

I

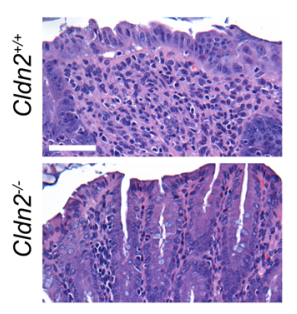

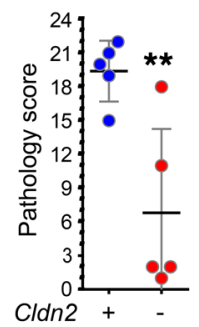

Figure 3. Claudin-2 knockout limits immune-mediated colitis severity. (A) Weight loss and (B) disease activity induced by T cell transfer are attenuated in $\mathrm{Cldn}^{-/-} \mathrm{Rag}^{-/-}$ mice (Cldn2 $2^{-/,}$, red circles), relative to that of $\mathrm{Cldn2}^{+/+} \mathrm{Rag1}^{-/-}$mice (Cldn2 ${ }^{+/+}$, blue circles). $n=9-10$ per genotype. Two-tailed $t$ test on day 56. (C) Consistent with reduced mucosal damage, barrier function was maintained in $\mathrm{Cldn2}^{-/-} \mathrm{Ragi}^{1 /-}$ mice relative to that of Cldn2 ${ }^{+/+}$Rag $^{-1-}$ mice. Dextran (4 kDa) flux was normalized to mice without $T$ cell transfer. $n=$ 8 per genotype. Two-tailed $t$ test. (D) Claudin-2 (CLDN2, green) expression is increased in proximal colon of $\mathrm{Cldn2}^{+/+} \mathrm{Rag}^{1 /-}$ mice on day 56. ZO-1 (red) expression is maintained in Cldn2 $2^{+/+}$ $\mathrm{Rag}^{1 /-}$ and $\mathrm{Cldn2}^{-/-} \mathrm{Rag}^{1 /-}$ mice. (E) Following $T$ cell transfer, fecal water content increased more rapidly in $\mathrm{Cldn2}^{+/+} \mathrm{Rag1}^{-/-}$mice relative to $\mathrm{Cldn}^{-1-}$ Rag1 $1^{-/-}$mice. $n=5$ per genotype. Two-tailed $t$ test on day 56. (F) Mucosal IFN- $\gamma$ and (G) TNF on day 56 after T cell transfer. $n$ $=5$ per genotype. Two-tailed $t$ test. (H) Representative immunostain of proximal colon showing CD3 (green) and E-cadherin (ECAD, red) and corresponding quantitative analysis. $\mathrm{T}$ cell recruitment is blunted in $\mathrm{Cldn2}^{-/-} \mathrm{Rag}^{-1 /}$ mice. $n=5-9$ per genotype. Two-tailed $t$ test. (I) Representative pathology scores on day 56. $n=5$ per genotype. Two-tailed $t$ test. Data presented in this figure are typical of 5 independent experiments. ${ }^{*} P<0.05$; ${ }^{* *} P<0.01$. Scale bars: $50 \mu \mathrm{m}$. sion of colitis. Colonic epithelial occludin expression was slightly, but significantly, reduced in colitic Cldn2 $2^{+/+} \operatorname{Rag1}^{-/-}$and $\mathrm{Cldn} 2^{-/-}$ Rag1 $1^{-1-}$ mice, relative to healthy controls, but there was no difference between genotypes (Figure $5 \mathrm{C}$ ).

To determine if insufficient luminal hydration was responsible for obstruction and mortality in $\mathrm{Cldn2}^{-/-} \mathrm{Rag1}^{-/-}$mice, polyethylene glycol was added to the drinking water in order to induce mild osmotic diarrhea. This prevented the development of intestinal obstruction in $\mathrm{Cldn}^{-/-} \mathrm{Rag1}^{-/-}$mice (Figure 5D). Polyethylene glycol treatment did not, however, affect claudin-15 expression (Figure 5E), suggesting that inadequate luminal hydration was not the stimulus for this upregulation. Disease severity and histopathology scores were unaffected by polyethylene glycol (Figure $5 \mathrm{~F}$ ), but survival of $\mathrm{Cldn}^{-/-} \mathrm{Rag1}^{-/-}$mice improved to equal that of Cldn2 ${ }^{+/+} \mathrm{Rag1}^{-/-}$mice (Figure $5 \mathrm{G}$ ). These data therefore demonstrate that claudin-2-mediated water efflux contributes significantly to diarrhea and that the resulting increases in luminal fluid prevent intestinal obstruction.

CK2 inhibition prevents claudin-2 function but does not affect DSS colitis. We have identified a series of molecular interactions between occludin, $\mathrm{ZO}-1$, and claudin- 2 that are regulated by casein kinase-2 (CK2) (50). CK2 normally phosphorylates S408 within the occludin C-terminal cytoplasmic tail, but CK2 inhibition allows dephosphorylation of this site (53). This enhances the affinity of occludin for the ZO-1 U5-GuK domain $(50,54)$. Binding of this bimolecular complex to the C-terminus of claudin-2 via the ZO-1 PDZ1 domain inactivates claudin-2 pore function (Figure
6A and ref. 50). In vitro, CK2 inhibition reversed IL-13-induced, claudin-2-mediated permeability increases (50). To determine if this regulatory pathway is active in vivo, mice were treated with IL-13 with or without a CK2 inhibitor. IL-13 robustly induced claudin-2 upregulation, and this was not affected by the CK2 inhibitor (Figure 6B). CK2 inhibition was, however, sufficient to completely block IL-13-induced increases in intestinal paracellular cation permeability (Figure 6C). Thus, CK2 inhibition can inactivate claudin-2 channels in vivo.

The efficacy of CK2 inhibition in blocking claudin-2 pore function suggested that this might be an effective therapy for experimental IBD. Previous work has, however, shown that CK2 is upregulated in DSS colitis and IBD and defined CK2 as a critical regulator of epithelial survival, proliferation, and migration in chronic intestinal inflammation (55). We were, therefore, concerned that systemic CK2 inhibition could exacerbate colitis. To address this, CK2 was inhibited using CX-4945, an orally bioavailable CK2 inhibitor (56-59). CK2 inhibition neither accelerated nor attenuated DSS-induced weight loss (Figure 6D) but caused a slight reduction in histopathology scores (Figure 6E). The effects of CK2 inhibition were identical in $\mathrm{Cldn2^{+/+ }}$ and $\mathrm{Cldn2^{-/ }}$ mice, indicating that they were independent of claudin-2 function. The data further indicate that CK2 is not essential for mucosal homeostasis and that CK2 inhibition may not have the in vivo toxicities predicted by previous in vitro studies.

CK2 inhibition attenuates immune-mediated, experimental $I B D$ via a claudin-2-dependent mechanism. As in human IBD 
A

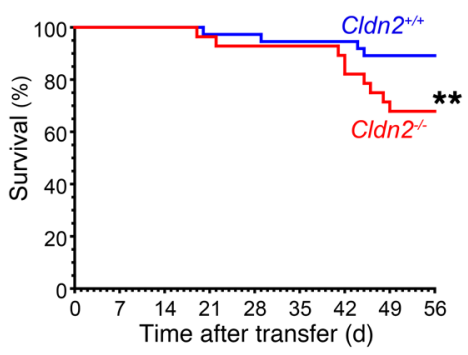

C

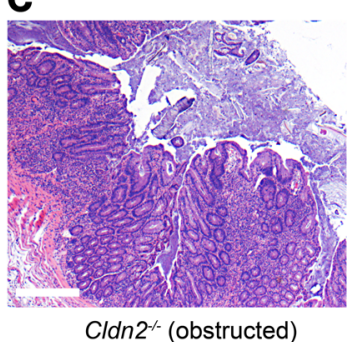

D

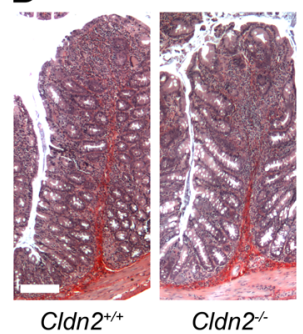

G
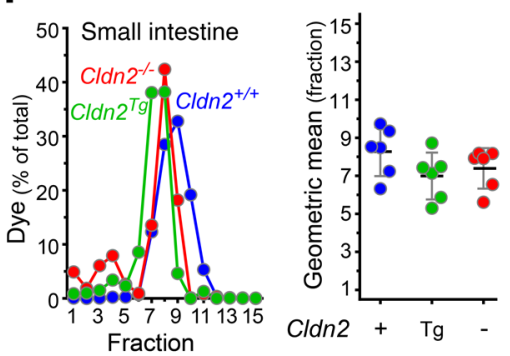

$\mathbf{F}$
B

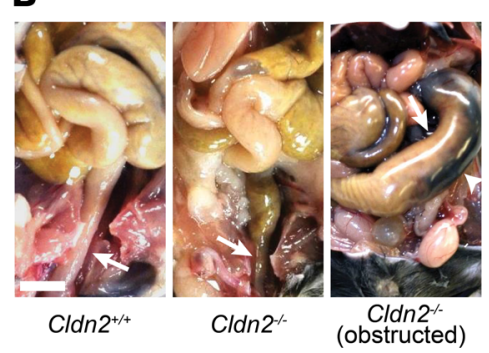

E

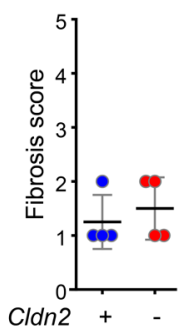

Figure 4. Despite reduced immune-mediated colitis severity, survival is compromised in

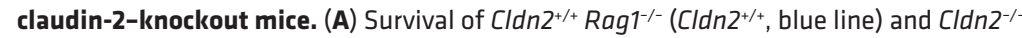

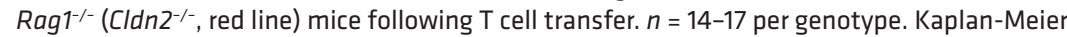
log-rank test. Data are representative of 5 independent experiments (B) Representative gross images of nonobstructed colons (arrows) of $\mathrm{Cldn2}^{+/+} \mathrm{Rag}^{-/-}$and $\mathrm{Cldn2}^{-/-} \mathrm{Rag}^{-/-}$mice, as well as obstructed (arrow) and ischemic (arrowhead) colon from a Cldn2 $2^{-1-} \mathrm{Rag}^{1 /-}$ mouse. Scale bar: $0.5 \mathrm{~cm}$. (C) Representative histopathology of obstructed Cldn2 $2^{-/-} \mathrm{Rag}^{1 /-}$ mouse

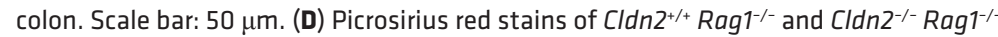
mouse colons on day 56. Scale bar: $50 \mu \mathrm{m}$. (E) Fibrosis scores of colons from Cldn2 $2^{+/+} \operatorname{Rag}^{1 /-}$ (blue circles) and Cldn2 $2^{-/-}$Rag1 $^{-1-}$ (red circles) mice on day 56. $n=4$ per genotype. Two-tailed $t$ test. (F) Small intestine motility in $\mathrm{Cldn}^{+/+}, \mathrm{Cldn} 2^{T_{g}}$ (green circles), and Cldn $2^{+/+}$mice assessed as dye content of each fraction and geometric mean of dye distribution. $n=6$ per genotype. ANOVA with Bonferroni's correction. (C) Colonic motility assessed as dye content of each fraction and geometric mean of dye distribution. $n=6$ per genotype. ANOVA with Bonferroni's correction. ${ }^{* *} P<0.01$.

(55), intestinal epithelial CK2 expression increased during immune-mediated experimental colitis (Figure 7A). The magnitude of this upregulation was similar in $\mathrm{Cldn2}^{-/-} \mathrm{Rag1}^{-/-}$and Cldn2 ${ }^{+/+} \mathrm{Rag1}^{-/-}$mice (Figure 7A). To assess the impact of CK2 upregulation, mice received a CK2 inhibitor by daily gavage. This treatment, which was not initiated until onset of clinically evident disease on day 10 after $\mathrm{T}$ cell transfer, markedly reduced disease severity, as indicated by weight loss (Figure 7B) and disease activity scores. This beneficial effect of CK2 inhibition was claudin-2 dependent, as the inhibitor was ineffective in $\mathrm{Cldn2^{-/- }}$ Rag1 $1^{-/-}$mice (Figure 7B). CK2 inhibition also limited increases in fecal water (Figure 7C) and $\mathrm{Na}^{+}$(Figure 7D) in Cldn2 $2^{+/+}$Rag1 $^{-/-}$ mice such that they were similar to $C l d n 2^{-/-} \operatorname{Ragl}^{-/-}$mice. This further suggests that CK2 inhibition reduced claudin-2 pore function in vivo. Importantly, despite causing the phenotype of $\mathrm{Cldn}^{+/+} \mathrm{Rag}^{1^{-/}}$ mice to mimic that of $\mathrm{Cldn2}^{-/-} \mathrm{Rag1}^{-/-}$mice, CK2 inhibition did not increase mortality of $\mathrm{Cldn} 2^{+/+}$ $\mathrm{Rag1}^{-/-}$mice (Figure 7E). CK2-inhibitor-induced claudin-2 channel inactivation was nevertheless sufficient to limit leak and unrestricted pathway barrier loss (Figure 7F), mucosal T cell infiltration (Figure 7G), and histopathologic progression (Figure $7 \mathrm{H}$ ) in $\mathrm{Cldn2}^{+/+} \mathrm{Rag1}^{-/-}$mice to levels observed in $\mathrm{Cldn2}^{-/-} \mathrm{Rag1}^{-/-}$mice. Although CK2 is widely expressed and known to be promiscuous with respect to substrates, the absence of apparent toxicities suggests that CK2 does not serve other critical functions in the context of immune-mediated experimental colitis. The dependence on claudin-2 expression indicates, however, that CK2 inhibition limits disease by inactivating claudin-2 channels.

\section{Discussion}

In contrast to macromolecular barrier loss, no studies have characterized the impact of size- and charge-selective permeability increases on immune-mediated colitis. In vitro studies have shown that claudin-2, which is upregulated in colitis, creates paracellular channels that accommodate water and $\mathrm{Na}^{+}$but not larger molecules. Here, we focused on the effects of in vivo claudin-2 expression on barrier function and immune-mediated disease. Claudin-2 upregulation was required for IL-13-induced increases in paracellular water and $\mathrm{Na}^{+}$permeability; these changes were recapitulated by transgenic claudin-2 overexpression in vivo. We then sought to determine whether claudin-2 upregulation in immune-mediated experimental IBD represents an adaptive, prohomeostatic response or, alternatively, enhances disease progression. The data indicate that claudin-2 upregulation promotes mucosal immune activation and increases experimental IBD severity. Conversely, we found that in vivo CK2 inhibition inactivates claudin-2 channels, prevents acute claudin-2-mediated paracellular permeability increases, and improves outcomes in experimental IBD.

Despite well-characterized size and charge selectivity in vitro $(11,12,14,15,43,46)$, the impact of claudin-2 expression on in vivo barrier function has been controversial $(27,40,41,60)$. In part, this debate reflects the coexistence of claudin-2 upregulation and increased macromolecular flux in colitis $(27,40)$. The idea that claudin-2 channels can accommodate macromolecules is also supported by a report that flux of 4 -kDa dextran (28 A diameter) was increased by transgenic human claudin-2 expression (41). In contrast, the transgenic mice described here displayed size- and charge-selective permeability increases that was limited to molecules with diameters less than 6-8 9 (29). The discordance between these different claudin-2-transgenic mice is puzzling, but cannot be due to the promoter used, as both studies 
A

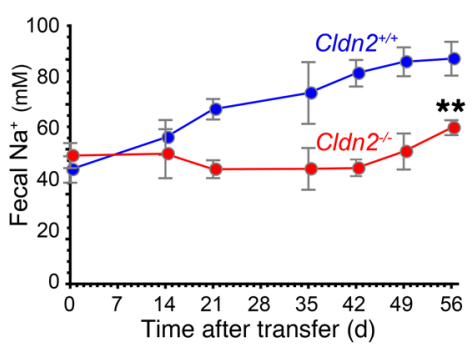

D

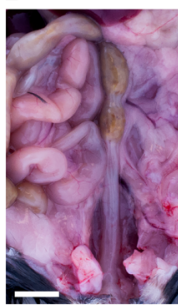

Cldn2 $2^{+/+}$

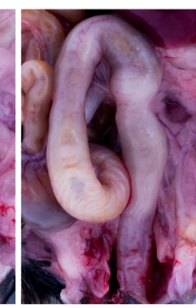

Cldn2-

$\mathbf{F}$
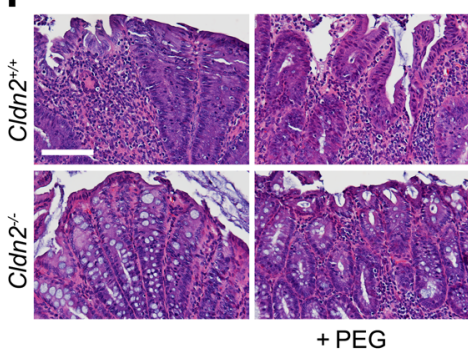

B

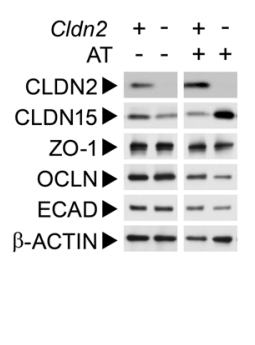

C

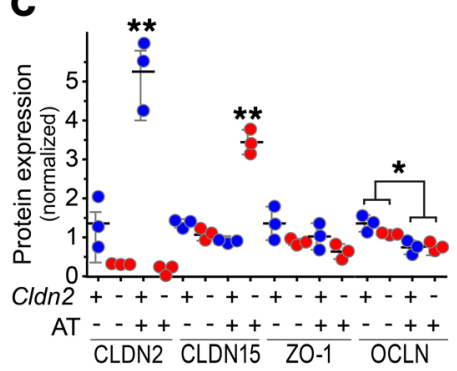

$\mathbf{E}$

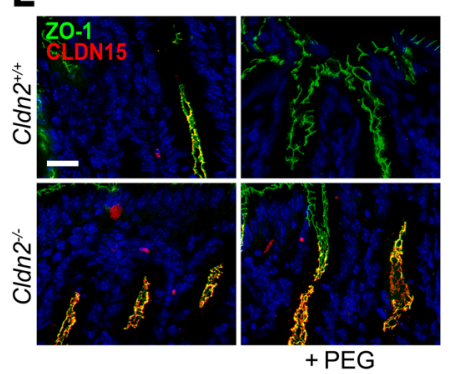

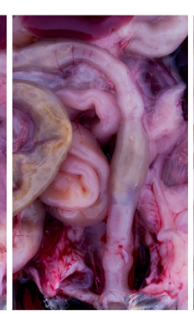

$\mathrm{Cldn} 2^{+*}$
$+\mathrm{PEG}$

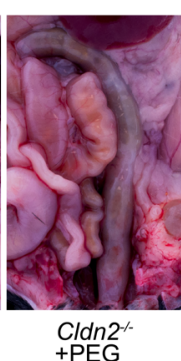

G

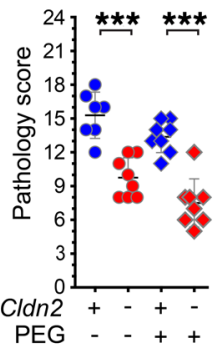

$\mathbf{G}$

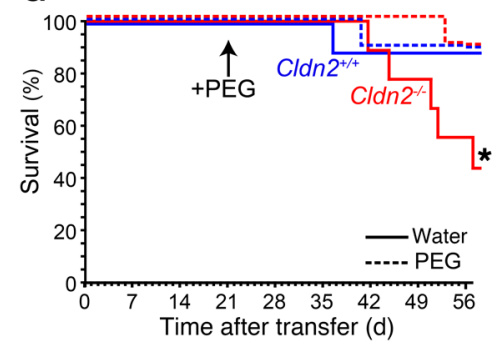

Figure 5. Insufficient luminal hydration leads to increased mortality in claudin-2-knockout mice. (A)

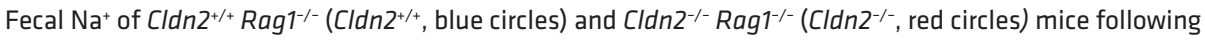
T cell transfer. Data are representative of 5 independent experiments. $n=6$ per genotype. Two-tailed $t$ test on day 56. (B) Immunoblots of isolated colonic epithelia from Cldn2 $2^{+/+} \operatorname{Rag}^{1 /-}$ and $\mathrm{Cldn}^{-/-} \mathrm{Rag}^{1 /-}$ mice without (-) or with (+) T cell transfer (AT) on day 56. Claudin-2 (CLDN2), claudin-15 (CLDN15), ZO-1, occludin (OCLN), E-cadherin (ECAD), and $\beta$-actin are shown. Due to the number of antigens probed, samples are not all from the same membrane but are from blots that were performed in parallel using the same samples. The data were validated further by quantitative densitometry. (C) Densitometry of immunoblots, as in B. $n=3$ per condition. ANOVA with Bonferroni's correction. Data are representative of 3 independent experiments. (D) Gross images of $\mathrm{Cldn2}^{+/+} \mathrm{Rag}^{1 /-}$ and $\mathrm{Cldn2^{-/- }} \mathrm{Rag}^{1 /-}$ mouse colons with polyethylene glycol (+PEG) or without PEG treatment on day 56. Scale bar: $0.5 \mathrm{~cm}$. (E) ZO-1 (green) and claudin-15 (red) expression in proximal colon of vehicle- and PEG-treated mice 56 days after T cell transfer. Scale bar: $20 \mu \mathrm{m}$. (F) Representative colonic pathology scores in $\mathrm{Cldn2}^{+/+} \mathrm{Rag}^{-/-}$and $\mathrm{Cldn2^{-/- }} \mathrm{Rag}^{-1 /-}$ mice without PEG (circles) or with PEG (diamonds) treatment on day 56. Scale bar: $50 \mu \mathrm{m} . n=7-8$ per genotype and condition. ANOVA

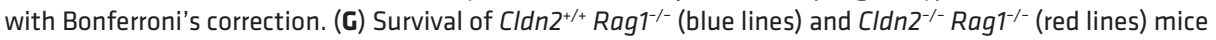
following provision of normal drinking water (solid lines) or water with PEG (dashed lines) beginning on day 21. $n=9-10$ per condition. Kaplan-Meier log-rank test. Data in $\mathbf{D}-\mathbf{C}$ are representative of 3 independent experiments. ${ }^{*} P<0.05 ;{ }^{* *} P<0.01 ;{ }^{* *} P<0.001$.

relied on the same 9-kb Vil1 promoter (61). It is notable that epithelial proliferation was increased, in the absence of any stimuli, in human claudin-2-transgenic but not mouse claudin-2-transgenic mice $(29,41)$. This suggests that low-grade epithelial damage may be present in human claudin-2-transgenic mice and that associated epithelial damage may explain the increased $4-\mathrm{kDa}$ dextran flux (41). Although it is possible that mouse claudin-2 function was modified by the N-terminal GFP tag, this is unlikely based on previous in vitro and in vivo analyses $(29,50,62,63)$. The permeability changes induced by in vivo overexpression of
GFP-tagged mouse claudin-2 therefore mirror those of in vitro claudin-2 overexpression $(12,14,15,43)$.

Strikingly, immune-mediated experimental IBD was far more severe in $\mathrm{Cldn}^{\mathrm{Tg}} \mathrm{Rag1}^{-1-}$ mice, while Cldn2 $2^{--}$ Rag1 $1^{-/}$mice were protected from colitis. This was not anticipated given that reciprocal results, i.e., protection by claudin-2 overexpression and exacerbation by claudin-2 deletion, were observed in chemical and infectious colitis $(29,41,42)$. In infection, osmotic diarrhea rescued Cldn $2^{--}$mice, indicating that claudin-2 promotes pathogen clearance by enhancing paracellular water efflux. Similarly, fecal water increases induced by transgenic claudin-2 overexpression (41) may dilute DSS within the distal colon to reduce mucosal injury in $\mathrm{Cldn} 2^{T_{g}}$ mice. Thus, claudin-2-mediated water efflux may explain the protective effects of claudin-2 upregulation in both chemical and infectious colitis.

Consistent with claudin-2-mediated luminal water efflux, insufficient luminal hydration explains the increased mortality of $\mathrm{Cldn}^{-/-} \mathrm{Rag}^{-/-}$ mice in immune-mediated experimental IBD; this increased mortality was eliminated by mild diarrhea. However, unlike infectious colitis, increased luminal hydration did not affect disease severity. This cannot, therefore, be the mechanism by which claudin-2 deletion reduces immune-mediated experimental IBD severity. One alternative explanation could be that claudin-2 expression increases, and claudin-2 knockout reduces, mucosal, i.e., lamina propria, $\mathrm{Na}^{+}(22)$. This idea is supported by previous work showing that luminal $\mathrm{Na}^{+}$, but not active transepithelial transport, is required to maintain villous lamina propria $\mathrm{Na}^{+}$ concentrations of up to $900 \mathrm{mM}$, corresponding to osmolarities of up to $1,600 \mathrm{mOsm}$ (64). Although villous lamina propria $\mathrm{Na}^{+}$concentrations have not been measured in claudin-2- or claudin-15-knockout mice, luminal $\mathrm{Na}^{+}$is reduced in these mice (22). Thus, claudin-2 deficiency could be expected to result in reduced luminal and villous lamina propria $\mathrm{Na}^{+}$concentrations in some situations. Together with previous work showing that increased extracellular $\mathrm{Na}^{+}$concentrations activate salt-sensitive serum-glucocorticoid kinase 1 (SGK1) to enhance Th1 and Th17 cell differentiation (65-67), these data support the hypothesis that claudin-2 deficiency reduces, and claudin- 2 over- 
A

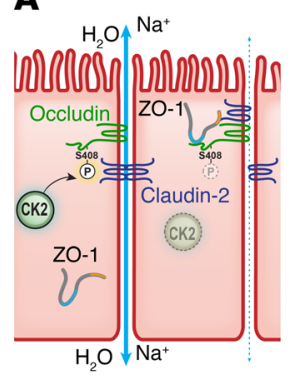

B

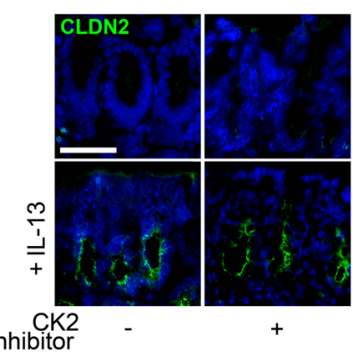

C

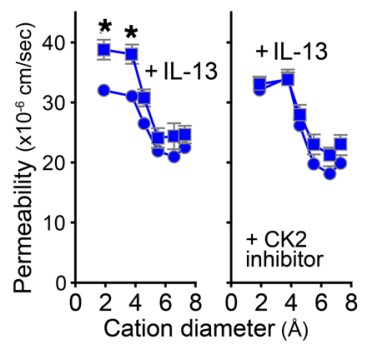

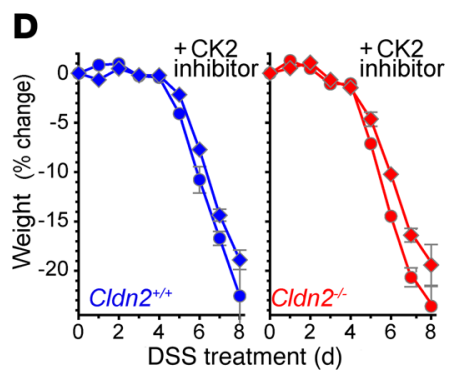

E

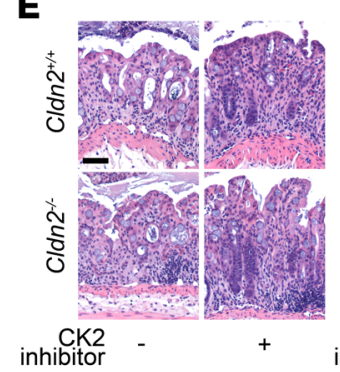

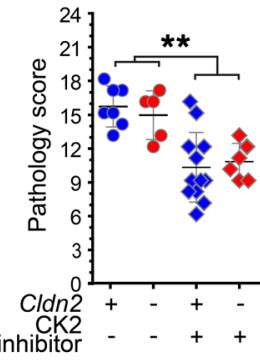

Figure 6. CK2 inhibition does not affect DSS colitis severity. (A) In vitro studies have shown that casein kinase-2 (CK2) inhibition results in occludin $\$ 408$ dephosphorylation. This increases occludin's affinity for ZO-1 and leads to assembly of a trimolecular complex composed of occludin, ZO-1, and claudin-2 that inactivates claudin-2 channels (50). (B) Claudin-2 (CLDN2, green) expression in proximal colon of WT mice treated with vehicle or IL-13 without or with CK2 inhibition. Nuclei (blue) are shown for reference. Data are representative of 3 independent experiments. (C) Ussing chamber analysis of proximal colonic mucosal permeability to $\mathrm{Na}^{+}$and 5 larger cations (methylamine, ethylamine, tetramethylammonium, tetraethylammonium, and $\mathrm{N}$-methyl-D-glucamine), as in Figure 1. Both graphs show $\mathrm{Cldn2}^{+/+}$mice treated with vehicle (circles) or IL-13 (squares). Mice that were treated with vehicle (left) or CK2 inhibitor (right) are shown in the 2 graphs. Data compiled from 3 independent experiments. ANOVA with Bonferroni's correction. (D) Weight change of $\mathrm{Cldn2}^{+/+}$and Cldn2 $2^{-/-}$mice following DSS treatment without (circles) or with (diamonds) CK2 inhibitor. $n=5-7$ per group. ANOVA with Bonferroni's correction. (E) Representative pathology scores on day 8 after DSS treatment. $n=5-12$ per group. ANOVA with Bonferroni's correction. Data in $\mathbf{D}$ and $\mathbf{E}$ are representative of 4 independent experiments. ${ }^{*} P<0.05$; ${ }^{* *} P<0.01$. Scale bars: $50 \mu \mathrm{m}$.

expression enhances, lamina propria $\mathrm{Na}^{+}$to drive pathogenic $\mathrm{T}$ cell development. Consistent with this, a high-salt diet is sufficient to increase mucosal Th17 cell frequency $(66,68)$ and to exacerbate DSS-induced chemical colitis $(68,69)$. Nevertheless, it remains unclear why claudin-15, which was upregulated in colitic Cldn2 $2^{-1-}$ Rag1 ${ }^{-/}$mice, was not sufficient to replace claudin-2 function and restore Th1 and Th17 cell differentiation. Further characterization of functional differences between claudin- 2 and claudin- 15 as well as their interactions with dietary $\mathrm{Na}^{+}$and effects on mucosal $\mathrm{Na}^{+}$ is, therefore, warranted along with exploration of the impact of claudin- 2 expression on tissue $\mathrm{Na}^{+}$concentrations and $\mathrm{T}$ cell differentiation in vivo.

We previously discovered a complex signaling pathway by which CK2 activity facilitates, and CK2 inhibition blocks, claudin-2 pore function (50). Although the responsible phosphatase has not been identified, in vitro analyses have shown that CK2 inhibition leads to occludin dephosphorylation at specific residues. Mutagenesis studies identified S408 within the occludin C-terminal tail as the key site which, when dephosphorylated, increases occludin affinity for the ZO-1 U5GuK domain. This bimolecular interaction increases anchoring, i.e., reduces fluo- rescence recovery after photobleaching (FRAP), of tight junction-associated occludin (50), and binding of this complex to claudin-2, via the ZO-1 PDZ1 domain, disrupts claudin-2 channel function. This can have profound functional consequences as, for example, CK2 inhibition is sufficient to acutely reverse claudin-2-mediated, IL-13-induced barrier loss in vitro (50).

Although CK2-dependent regulation of occludin S408 phosphorylation has not been confirmed in vivo, we previously used intravital imaging and FRAP analysis of transgenic GFPoccludin mice to demonstrate that CK2 inhibition increases occludin anchoring at the tight junction in vivo (50). Here, we have shown that the second part of this signal transduction mechanism, claudin-2 channel inactivation, also occurs in vivo. Importantly, the effect of CK2 inhibition was only apparent after claudin-2 upregulation and, characteristic of claudin-2 channels, was selective for $\mathrm{Na}^{+}$and methylamine cations.

To determine if pharmacological claudin-2 channel inactivation could be beneficial in chronic immune-mediated colitis, mice were dosed with a highly specific, orally bioavailable CK2 inhibitor that was well tolerated in both preclinical studies and phase I clinical trials (70, 71). Consistent with claudin-2 channel inactivation, inhibitor treatment reduced fecal water and $\mathrm{Na}^{+}$ increases during disease progression. This was claudin-2 dependent, as CK2 inhibition did not affect fecal water and $\mathrm{Na}^{+}$in $\mathrm{Cldn}^{-/-} \mathrm{Rag}^{-/-}$mice. Moreover, CK2 inhibition attenuated weight loss, $\mathrm{T}$ cell recruitment, and histopathology in $\mathrm{Cldn2^{+/+ }}$ Rag1 $1^{--}$mice to levels seen in $\mathrm{Cldnn}^{-/-} \mathrm{Rag1}^{-/-}$mice. Again, this was claudin-2 dependent; CK2 inhibition did not benefit $\mathrm{Cldnn}^{-/-} \mathrm{Rag1}^{-/}$mice. Thus, despite the promiscuity of CK2, the data indicate that the effects of CK2 inhibition are primarily due to claudin-2 channel inactivation. In contrast to claudin-2 knockout, however, CK2 inhibition was not accompanied by intestinal obstruction or increases in mortality. This or similar approaches may therefore be safe for clinical use.

As a whole, our data indicate that epithelial claudin-2 upregulation promotes progression of immune-mediated experimental IBD. This contrasts sharply with infectious and chemical colitis, suggesting that claudin-2 pore function has pleiotropic effects on intestinal mucosal pathophysiology. The data also demonstrate that CK2 inhibition inactivates claudin-2 pores in vivo and, predominantly via this mechanism, limits colitis progression. Although the broad expression and multiple functions of CK2 may limit utility of CK2 inhibition in colitis, we anticipate that further molecular definition of claudin-2 channel function (14) will lead to development of more precisely targeted therapies.

\section{Methods}

Mice. C57BL/6J mice (stock 000664) and Rag1/- (B6.129S7Rag1 ${ }^{\text {tmIMom }} / \mathrm{J}$ ) mice (stock 002216) were purchased from The Jackson 
A

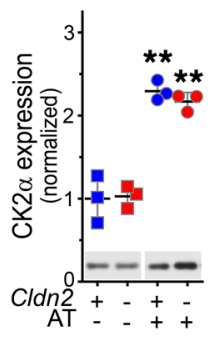

B

C

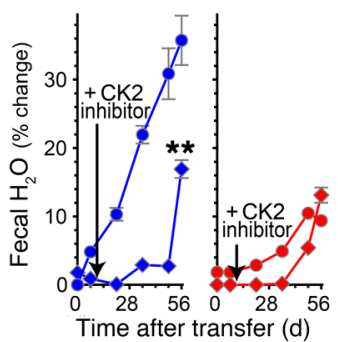

D

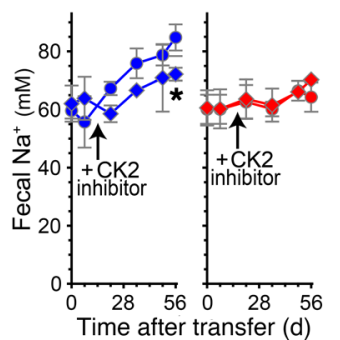

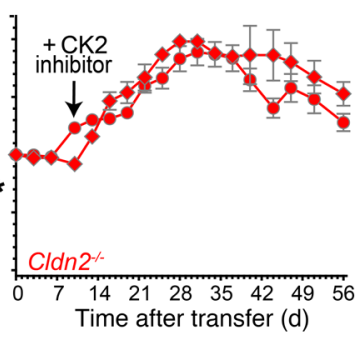

E

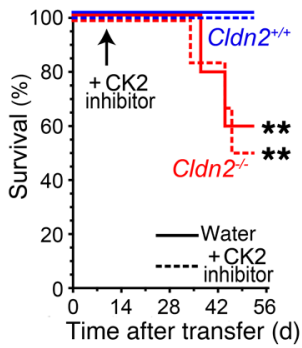

$\mathbf{F}$

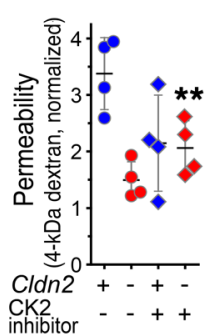

G

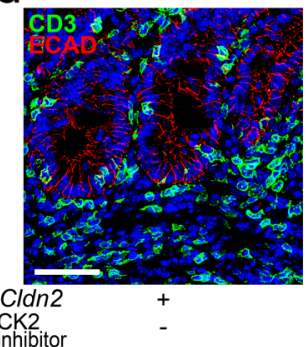

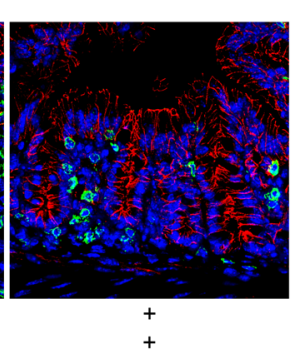
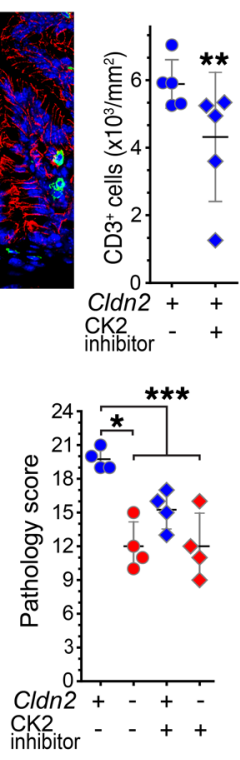

inhibitor $-\quad+$
Figure 7. CK2 inhibition limits immune-mediated colitis severity via a claudin-2-dependent mechanism. (A) Immunoblots of the catalytic $\alpha$ subunit of casein kinase-2 (CK2) in isolated colonic epithelia from Cldn2 $2^{+/+} \mathrm{Rag1}^{-/-}\left(\mathrm{CldnZ}^{+/+}\right.$, blue

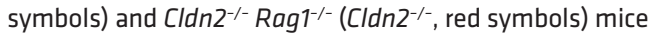
without adoptive T cell transfer (+AT) (squares) or on day 56 after transfer (circles). $n=3$ per condition. Data are representative of 3 independent experiments. ANOVA with Bonferroni's correction. (B) Weight loss following T cell transfer was attenuated by CK2 inhibition in Cldn2 ${ }^{+/+} \mathrm{Rag}^{1^{-/}}$mice (left graph) but not $\mathrm{Cldn2}^{-/-} \mathrm{Rag1}^{-/-}$mice (right graph). Mice were treated with vehicle (circles) or CK2 inhibitor (diamonds), beginning 10 days after T cell transfer. $n=5-6$ per condition. ANOVA with Bonferroni's correction on day 56. (C) Fecal water and (D) $\mathrm{Na}^{+}$content following $\mathrm{T}$ cell transfer were reduced in

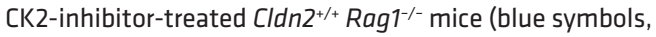
left graphs); there was no effect in $\mathrm{Cldn2}^{-{ }^{-/}} \mathrm{Rag}^{-1-}$ mice (red symbols, right graphs). $n=5-6$ per condition. ANOVA with Bonferroni's correction on day 56. (E) Survival of $\mathrm{Cldn2^{+/+ }}$ Rag $^{-1-}$ and Cldn2 $2^{-/-}$Rag $^{1 /-}$ mice following T cell transfer without (solid lines) or with (dashed lines) CK2 inhibitor treatment. $n=5-6$ per condition. Kaplan-Meier log-rank test. (F) Intestinal barrier function on day 56 after T cell transfer was preserved in $\mathrm{CK} 2$-inhibitor-treated $\mathrm{Cldn2}^{+/+} \mathrm{Rag}^{1 /-}$ mice. $n=4$ per condition. ANOVA with Bonferroni's correction. (G) Immunostain of CD3 (green) and E-cadherin (ECAD, red). CK2 inhibition reduced $T$ cell recruitment into proximal colonic mucosae of $\mathrm{Cldn}^{+/+} \mathrm{Rag}^{-/-}$mice. Scale bar: $50 \mu \mathrm{m} . n=5$ per condition. ANOVA with Bonferroni's correction. (H) Histopathology and scores on day 56 after $T$ cell transfer without or with CK2 inhibitor treatment. Scale bar: $50 \mu \mathrm{m} . n=4$ per genotype and condition. ANOVA with Bonferroni's correction. Data presented in $\mathbf{B}-\mathbf{H}$ are typical of 3 independent experiments. ${ }^{*} P<0.05 ;{ }^{* *} P<0.01 ;{ }^{* *} P<0.001$.
H

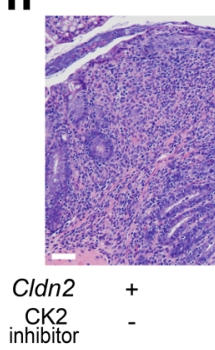

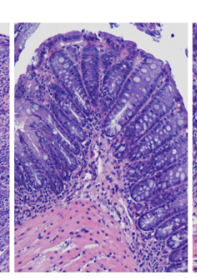
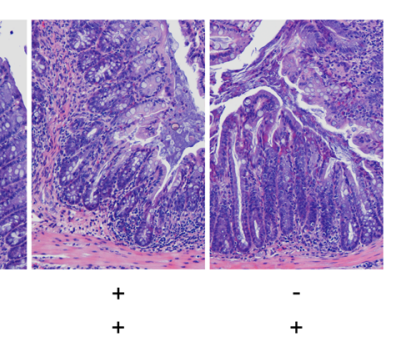

Laboratory. Claudin-2-knockout mice on a C57BL/6J background have been described previously $(4,29)$. Transgenic GFP-claudin-2 mice were generated using the 9-kb Vil1 promoter $(29,72)$. Mice were bred under specific pathogen-free conditions and used at 6 to 8 weeks of age. Littermates or cohoused mice were used for all experiments. Individual experiments were segregated, but all studies were performed in both sexes.

Cytokine and CK2 inhibitor treatment. Mice were injected i.p. with 1.0 to $2.5 \mu \mathrm{g}$ (160 units) of recombinant murine IL-13 (R\&D Systems) 14 hours before tissue harvest. Mice were injected i.p. with the CK2 inhibitor TBCA [(E)-3-(2,3,4,5-tetrabromophenyl)acrylic acid, $50 \mathrm{mg} /$ kg; MilliporeSigma] 24 hours before and 2 hours after IL-13 injection. The CK2 inhibitor CX-4945 (provided by Cylene Pharmaceuticals) was delivered by gavage twice daily at $75 \mathrm{mg} / \mathrm{kg}$ in DSS experiments. Because $\mathrm{T}$ cell transfer colitis experiments required treatment over much longer intervals, mice were gavaged only once each day, begin- ning 10 days after transfer. In an effort to compensate for the reduced treatment frequency, the drug dose was increased to $100 \mathrm{mg} / \mathrm{kg}$.

Colitis induction. Acute colitis was induced by adding 3\% DSS (MilliporeSigma) to drinking water and following mice over 8 days (35). Immune-mediated colitis was induced in immunodeficient Rag1 $1^{-/}$mice by transfer of $5 \times 10^{5}$ purified $\mathrm{CD} 4^{+} \mathrm{CD} 45 \mathrm{Rb} \mathrm{R}^{\text {hi }} \mathrm{T}$ cells, as described previously $(33,35)$. Disease activity was scored from 0 to 2 each for motor activity, fur texture, posture, and diarrhea, for a total score of $0-8$, as described previously $(33,35)$.

Electrophysiology. Stripped proximal colonic mucosa was mounted in $0.3-\mathrm{cm}^{2}$ surface area Ussing chambers (Physiologic Instruments) containing $135 \mathrm{mM} \mathrm{NaCl}, 5 \mathrm{mM} \mathrm{KOH}, 1.3 \mathrm{mM} \mathrm{CaCl}_{2}, 0.4 \mathrm{mM} \mathrm{MgSO}_{4}$, $0.3 \mathrm{mM} \mathrm{NaH}_{2} \mathrm{PO}_{4}, 15 \mathrm{mM}$ HEPES, $5.5 \mathrm{mM}$ D-glucose, $\mathrm{pH}$ 7.4, and bubbled continuously with $100 \% \mathrm{O}_{2}$ at $37^{\circ} \mathrm{C}$. Resistance was calculated based on the potential generated by $10-\mu \mathrm{A}$ square-wave pulses. $\mathrm{NaCl}$ dilution potentials were measured as reported previously (43). 
Bi-ionic potentials were measured by replacing $\mathrm{Na}^{+}$(1.9 $\AA$ diameter) with methylamine $(3.8 \AA)$, ethylamine $(4.6 \AA)$, tetramethylammonium (5.6 ̊), tetraethylammonium (6.6 ̊), or $N$-methyl-D-glucamine (7.4 $\AA)$, as described previously $(15,50)$.

Intestinal permeability, motility, and fecal analyses. Mice were denied access to food but allowed water for 3 hours before gavage with $0.15 \mathrm{~mL}$ saline containing $12 \mathrm{mg}$ FITC-4-kDa dextran (MilliporeSigma). Serum was collected 4 hours later and analyzed as described previously (34). Motility was determined using FITC-70-kDa dextran (73). Fecal $\mathrm{Na}^{+}$ and water content were determined as described previously (29).

ELISA. Portions of colon $(0.5 \mathrm{~cm})$ were washed and homogenized in Bio-Plex buffers (Bio-Rad) as described previously (34). After freeze-thaw and sonication, supernatants ( 5 minutes at 4,500 $g$ ) were analyzed for total protein using a Bradford assay (Bio-Rad) and cytokines using Ready-SET-Go! ELISA kits (eBioscience).

Antibodies.Primaryantibodiesusedweremousemonoclonalanti-GFP, GFP-G1, Developmental Studies Hybridoma Bank, RRID AB_2619561; rabbit polyclonal anti-claudin-2 (MH44), 51-6100, Thermo Fisher Scientific, RRID AB_2533911; rabbit polyclonal anti-claudin-2, HPA051548, Atlas Antibodies, RRID AB_2681530; monoclonal mouse anti-claudin-2 (12H12), 32-5600, Thermo Fisher Scientific, RRID AB_2533085; rabbit monoclonal anti-claudin-4, ab210796, Abcam, RRID AB_2732879; rabbit polyclonal anti-claudin-15, 38-9200, Thermo Fisher Scientific, RRID AB_2533391; rat monoclonal anti-occludin (7C2C3), RRID AB_2819193; mouse monoclonal anti-occludin (OC-3F10), 33-1500, Thermo Fisher Scientific, RRID AB_2533101; rat monoclonal anti-ZO-1 (R40.76), RRID AB_2783859; rabbit monoclonal anti-E-cadherin, 3195, Cell Signaling Technology, RRID AB_2291471; mouse monoclonal anti-E-cadherin, 76055, Abcam, RRID AB_1310159; rabbit polyclonal anti-CK2 $\alpha$, ab10466, Abcam, RRID AB_297210; mouse monoclonal anti- $\beta$ actin, A1978, MilliporeSigma, RRID AB_476692; and rabbit monoclonal anti-CD3 (SP7), ab16669, Abcam, RRID AB_44342. Secondary antibodies used were IRDye 800CW-goat anti-rabbit IgG, 925-32211, LI-COR Biosciences, RRID AB_2651127; IRDye 680LT-goat antirabbit IgG, 926-68021, LI-COR Biosciences, RRID AB_10706309; IRDye 680RD-goat anti-mouse IgG, 925-68070, LI-COR Biosciences, RRID: AB_2651128; IRDye 800CW-goat anti-mouse IgG, 926-32210, LI-COR Biosciences, RRID AB_621842; HRP-goat antirabbit IgG, 7074, Cell Signaling Technology, RRID AB_2099233; HRP-horse anti-mouse IgG, 7076, Cell Signaling Technology RRID AB_330924; Alexa Fluor 594-donkey anti-rabbit IgG highly crossadsorbed $\mathrm{F}\left(\mathrm{ab}^{\prime}\right)_{2}$ fragments, 711-586-152, Jackson ImmunoResearch, RRID AB_2340622; Alexa Fluor 488-donkey anti-mouse IgG highly cross-adsorbed $\mathrm{F}\left(\mathrm{ab}^{\prime}\right)_{2}$ fragments, 715-546-151, Jackson ImmunoResearch RRID AB_2340850; and Alexa Fluor 488-donkey anti-rat IgG highly cross-adsorbed $\mathrm{F}\left(\mathrm{ab}^{\prime}\right)_{2}$ fragments, 712-546-153, Jackson ImmunoResearch RRID AB_2340686.

Epithelial cell isolation and Western blot. Colonic epithelial cells were isolated as described previously $(74,75)$. Cell lysates were separated by SDS-PAGE, transferred to PVDF membranes, and incubated with primary antibodies. Secondary antibodies were conjugated to HRP or infrared dyes, and proteins were detected using HyBlotCL film or an Odyssex Fc imager (LI-COR), respectively. Quantification used Image (NIH) and Image Studio (LI-COR) software.

Histological and immunofluorescence staining and microscopy. Colon segments were snap-frozen or formalin-fixed and paraffin-embedded (FFPE) as described previously $(29,34)$. H\&E and picrosirius red (76) staining was performed on $5-\mu \mathrm{m}$ paraffin sections. Sections $(5 \mu \mathrm{m})$ of snap-frozen tissue were fixed in 1\% PFA before permeabilization, blocking, and staining, as described previously $(34,75)$. Sections $(5 \mu \mathrm{m})$ of paraffin-embedded tissue microarrays were deparaffinized, epitopes unmasked, and autofluorescence quenched as described previously (34).

$\mathrm{H} \& \mathrm{E}$ - and picrosirius red-stained tissues were imaged using a DMLB microscope (Leica) with 10× HC FL PLAN NA 0.25, 20× HC FL PLAN FLUOTAR NA 0.5, and 40× HC FL PLAN NA 0.65 objectives and a MicroPublisher 3.3 CCD camera (QImaging) controlled by QCapture Pro 7.

Fluorescence micrographs were collected as stacks at $0.2-\mu \mathrm{m}$ intervals using an Axioplan 2 (Zeiss) with Chroma single-channel ET filter sets, $20 \times$ Plan-Apochromat NA 0.8 or $63 \times$ Plan-Apochromat NA 1.4 immersion objective, and a Coolsnap HQ camera controlled by MetaMorph 7.8 (Molecular Devices). Images were deconvoluted using Autoquant X3 (MediaCybernetics).

Histopathological scoring. Histopathological analysis of colitis was performed by a pathologist blinded to the experimental conditions. Colon tissues were scored on a scale of 0-3 for 8 parameters, yielding a maximum score of 24 . Scoring parameters were goblet cell depletion, mucosal hyperplasia, crypt cell apoptosis, epithelial erosion, lymphocytic infiltrate, polymorphonuclear (PMN) leukocyte infiltrate, crypt architectural distortion, and involvement of the submucosa. Fibrosis was scored on a 5-point scale on the basis of picrosirius red staining.

Statistics. For all data shown, specific numbers of mice in each group are indicated in figure legends. Data are presented as mean \pm SD. All data are representative of at least 3 independent experiments. Statistical significance was determined by 2-tailed Student's $t$ test, ANOVA with Bonferroni's correction, 2-tailed Mann-Whitney $U$ test, or Kaplan-Meier log-rank test, as indicated in the figure legends. Results with $P$ less than 0.05 were considered significant. In figures, ${ }^{*} P<0.05,{ }^{* *} P<0.01,{ }^{* * *} P<0.001$.

Study approval. All studies were approved by Institutional Animal Care and Use Committees at Brigham and Women's Hospital, Boston Children's Hospital, and the University of Chicago.

\section{Author contributions}

The project was conceived by JRT. PR, NS, PYT, PP, SCP, PRS, WTK, GS, and JRT performed experiments. ST provided claudin-2-knockout mice. Figures were prepared by PR, NS, PP, and JRT. The manuscript was written by JRT and revised by PR and JRT with input from all authors. PR and NS contributed equally to this work and are listed alphabetically.

\section{Acknowledgments}

This work was supported by Department of Defense grant PR181271 (to JRT), NIH grants R01DK61931 (to JRT), R01DK68271 (to JRT), and R24DK099803 (to JRT), the Harvard Digestive Disease Center (P30DK034854), and Crohn's and Colitis Foundation Research Fellowship Award 622459 (to NS). We thank Tiffany S. Davanzo (Slaybaugh Studios) for her beautiful illustrations, Heather Marlatt (Nationwide Histology) for her outstanding assistance with staining and tissue microarray development, and, alphabetically, Marion France (Brigham and Women's Hospital and Harvard Medical School), Ekaterina Khramtsova (University of Chicago), Steven Nilsen (Brigham and Women's Hospital and Harvard Medical School), Lora M.D.M. Ong (Brigham and Women's Hospital and Har- 
vard Medical School), Sheng-Ru Shiou (University of Chicago), Christopher Weber (University of Chicago), and Sunil Yeruva (Brigham and Women's Hospital and Harvard Medical School) for their technical contributions to this work.
Address correspondence to: Jerrold R. Turner, Brigham and Women's Hospital, Harvard Medical School, 77 Avenue Louis Pasteur, HNRB 730B, Boston, Massachusetts 02115, USA. Phone: 617.525.8165; Email: jrturner@bwh.harvard.edu.
1. Tisher CC, Yarger WE. Lanthanum permeability of the tight junction (zonula occludens) in the renal tubule of the rat. Kidney Int. 1973;3(4):238-250.

2. Marcial MA, Carlson SL, Madara JL. Partitioning of paracellular conductance along the ileal crypt-villus axis: a hypothesis based on structural analysis with detailed consideration of tight junction structure-function relationships. J Membr Biol. 1984;80(1):59-70.

3. Pei L, et al. Paracellular epithelial sodium transport maximizes energy efficiency in the kidney. JClin Invest. 2016;126(7):2509-2518.

4. Wada M, Tamura A, Takahashi N, Tsukita S. Loss of claudins 2 and 15 from mice causes defects in paracellular $\mathrm{Na}^{+}$flow and nutrient transport in gut and leads to death from malnutrition. Gastroenterology. 2013;144(2):369-380.

5. Farquhar MG, Palade GE. Junctional complexes in various epithelia. JCell Biol. 1963;17:375-412.

6. Machen TE, Erlij D, Wooding FB. Permeable junctional complexes. The movement of lanthanum across rabbit gallbladder and intestine. J Cell Biol. 1972;54(2):302-312.

7. Turner JR. Intestinal mucosal barrier function in health and disease. Nat Rev Immunol. 2009;9(11):799-809.

8. Anderson JM, Van Itallie CM. Physiology and function of the tight junction. Cold Spring Harb Perspect Biol. 2009;1(2):a002584.

9. Shen L, Weber CR, Raleigh DR, Yu D, Turner JR. Tight junction pore and leak pathways: a dynamic duo. Annu Rev Physiol. 2011;73:283-309.

10. Colegio OR, Van Itallie CM, McCrea HJ, Rahner C, Anderson JM. Claudins create chargeselective channels in the paracellular pathway between epithelial cells. Am JPhysiol, Cell Physiol. 2002;283(1):C142-C147.

11. Rosenthal R, et al. Claudin-2, a component of the tight junction, forms a paracellular water channel. JCell Sci. 2010;123(pt 11):1913-1921.

12. Amasheh S, et al. Claudin-2 expression induces cation-selective channels in tight junctions of epithelial cells. J Cell Sci. 2002;115(pt 24):4969-4976.

13. Rosenthal $\mathrm{R}$, et al. Claudin-15 forms a water channel through the tight junction with distinct function compared to claudin-2. Acta Physiol (Oxf). 2020;228(1):e13334.

14. Weber CR, et al. Claudin-2-dependent paracellular channels are dynamically gated. Elife. 2015;4:e09906.

15. Yu AS, et al. Molecular basis for cation selectivity in claudin-2-based paracellular pores: identification of an electrostatic interaction site. J Gen Physiol. 2009;133(1):111-127.

16. Rosenthal R, Günzel D, Krug SM, Schulzke JD, Fromm M, Yu AS. Claudin-2-mediated cation and water transport share a common pore. Acta Physiol (Oxf). 2017;219(2):521-536.

17. Kiuchi-Saishin Y, Gotoh S, Furuse M, Takasuga A, Tano Y, Tsukita S. Differential expression patterns of claudins, tight junction membrane proteins, in mouse nephron segments. J Am Soc Nephrol. 2002;13(4):875-886.

18. Escaffit F, Boudreau F, Beaulieu JF. Differential expression of claudin-2 along the human intestine: Implication of GATA- 4 in the maintenance of claudin-2 in differentiating cells. JCell Physiol. 2005;203(1):15-26.

19. Aung PP, Mitani Y, Sanada Y, Nakayama H, Matsusaki K, Yasui W. Differential expression of claudin-2 in normal human tissues and gastrointestinal carcinomas. Virchows Arch. 2006;448(4):428-434.

20. Uhlén M, et al. Proteomics. Tissue-based map of the human proteome. Science. 2015;347(6220):1260419.

21. Muto S, et al. Claudin-2-deficient mice are defective in the leaky and cation-selective paracellular permeability properties of renal proximal tubules. Proc Natl Acad Sci U S A. 2010;107(17):8011-8016.

22. Tamura A, et al. Loss of claudin-15, but not claudin-2, causes $\mathrm{Na}^{+}$deficiency and glucose malabsorption in mouse small intestine. Gastroenterology. 2011;140(3):913-923.

23. Alberini G, Benfenati F, Maragliano L. A refined model of claudin-15 tight junction paracellular architecture by molecular dynamics simulations. PLoS One. 2017;12(9):e0184190.

24. Tamura A, et al. Megaintestine in claudin-15-deficient mice. Gastroenterology. 2008;134(2):523-534.

25. Holmes JL, Van Itallie CM, Rasmussen JE, Anderson JM. Claudin profiling in the mouse during postnatal intestinal development and along the gastrointestinal tract reveals complex expression patterns. Gene Expr Patterns. 2006;6(6):581-588.

26. Ong MLDM, Yeruva S, Sailer A, Nilsen SP, Turner JR. Differential regulation of claudin-2 and claudin- 15 expression in children and adults with malabsorptive disease. Lab Invest. 2020;100(3):483-490.

27. Luettig J, Rosenthal R, Barmeyer C, Schulzke JD. Claudin-2 as a mediator of leaky gut barrier during intestinal inflammation. Tissue Barriers. 2015;3(1-2):e977176.

28. Schumann M, et al. Defective tight junctions in refractory celiac disease. Ann N Y Acad Sci. 2012;1258:43-51.

29. Tsai PY, et al. IL-22 upregulates epithelial claudin-2 to drive diarrhea and enteric pathogen clearance. Cell Host Microbe. 2017;21(6):671-681.e4.

30. Heller F, et al. Interleukin-13 is the key effector Th2 cytokine in ulcerative colitis that affects epithelial tight junctions, apoptosis, and cell restitution. Gastroenterology. 2005;129(2):550-564.

31. Prasad S, et al. Inflammatory processes have differential effects on claudins 2, 3 and 4 in colonic epithelial cells. Lab Invest. 2005;85(9):1139-1162.

32. Weber CR, Nalle SC, Tretiakova M, Rubin DT, Turner JR. Claudin-1 and claudin-2 expression is elevated in inflammatory bowel disease and may contribute to early neoplastic transformation.
Lab Invest. 2008;88(10):1110-1120.

33. Graham WV, et al. Intracellular MLCK1 diversion reverses barrier loss to restore mucosal homeostasis. Nat Med. 2019;25(4):690-700.

34. Nalle SC, et al. Graft-versus-host disease propagation depends on increased intestinal epithelial tight junction permeability. J Clin Invest. 2019;129(2):902-914.

35. Su L, et al. TNFR2 activates MLCK-dependent tight junction dysregulation to cause apoptosismediated barrier loss and experimental colitis. Gastroenterology. 2013;145(2):407-415.

36. Suzuki M, et al. Myosin light chain kinase expression induced via tumor necrosis factor receptor 2 signaling in the epithelial cells regulates the development of colitis-associated carcinogenesis. PLoS One. 2014;9(2):e88369.

37. Yi Z, Fan H, Liu X, Tang Q, Zuo D, Yang J. Adrenomedullin improves intestinal epithelial barrier function by downregulating myosin light chain phosphorylation in ulcerative colitis rats. Mol Med Rep. 2015;12(3):3615-3620.

38. Laukoetter MG, et al. JAM-A regulates permeability and inflammation in the intestine in vivo. JExp Med. 2007;204(13):3067-3076.

39. Vetrano S, et al. Unique role of junctional adhesion molecule-a in maintaining mucosal homeostasis in inflammatory bowel disease. Gastroenterology. 2008;135(1):173-184.

40. Bird L. T cells: IL-9 breaks down barriers. Nat Rev Immunol. 2014;14(7):432.

41. Ahmad R, et al. Targeted colonic claudin-2 expression renders resistance to epithelial injury, induces immune suppression, and protects from colitis. Mucosal Immunol. 2014;7(6):1340-1353.

42. Nishida M, Yoshida M, Nishiumi S, Furuse M, Azuma T. Claudin-2 regulates colorectal inflammation via myosin light chain kinase-dependent signaling. Dig Dis Sci. 2013;58(6):1546-1559.

43. Weber CR, et al. Epithelial myosin light chain kinase activation induces mucosal interleukin-13 expression to alter tight junction ion selectivity. J Biol Chem . 2010;285(16):12037-12046.

44. Günzel D, Yu AS. Claudins and the modulation of tight junction permeability. Physiol Rev. 2013;93(2):525-569.

45. Li J, Angelow S, Linge A, Zhuo M, Yu AS. Claudin-2 pore function requires an intramolecular disulfide bond between two conserved extracellular cysteines. Am J Physiol, Cell Physiol. 2013;305(2):C190-C196.

46. Van Itallie CM, et al. The density of small tight junction pores varies among cell types and is increased by expression of claudin-2. J Cell Sci. 2008;121(pt 3):298-305.

47. Angelow S, Yu AS. Structure-function studies of claudin extracellular domains by cysteinescanning mutagenesis. J Biol Chem. 2009;284(42):29205-29217.

48. Van Itallie CM, Fanning AS, Anderson JM. Reversal of charge selectivity in cation or 
anion-selective epithelial lines by expression of different claudins. Am J Physiol Renal Physiol. 2003;285(6):F1078-F1084.

49. Hou J, Rajagopal M, Yu AS. Claudins and the kidney. Annu Rev Physiol. 2013;75:479-501.

50. Raleigh DR, et al. Occludin S408 phosphorylation regulates tight junction protein interactions and barrier function. J Cell Biol. 2011;193(3):565-582.

51. Leach MW, Bean AG, Mauze S, Coffman RL, Powrie F. Inflammatory bowel disease in C.B-17 scid mice reconstituted with the CD45RB high subset of CD $4^{+}$T cells. Am J Pathol. 1996;148(5):1503-1515.

52. Krug SM, et al. Charge-selective claudin channels. Ann N Y Acad Sci. 2012;1257:20-28.

53. Cordenonsi M, et al. Xenopus laevis occludin. Identification of in vitro phosphorylation sites by protein kinase CK2 and association with cingulin. Eur J Biochem. 1999;264(2):374-384.

54. Dörfel MJ, et al. CK2-dependent phosphorylation of occludin regulates the interaction with ZO-proteins and tight junction integrity. Cell Commun Signal. 2013;11(1):40.

55. Koch S, Capaldo CT, Hilgarth RS, Fournier B, Parkos CA, Nusrat A. Protein kinase CK2 is a critical regulator of epithelial homeostasis in chronic intestinal inflammation. Mucosal Immunol. 2013;6(1):136-145.

56. Borad MJ, et al. A phase IB study of CX-4945 in combination with gemcitabine plus cisplatin in the frontline systemic treatment of patients with advanced cholangiocarcinoma. J Clin Oncol. 2017;35(4_suppl):294.

57. Marschke RF, et al. Findings from the phase I clinical trials of CX-4945, an orally available inhibitor of CK2.JClin Oncol.2011;29(15_suppl):3087.
58. Martins LR, et al. Activity of the clinical-stage CK2-specific inhibitor CX-4945 against chronic lymphocytic leukemia. Leukemia. 2014;28(1):179-182.

59. Jung $M$, et al. Inhibiting casein kinase 2 overcomes paclitaxel resistance in gastric cancer. Gastric Cancer. 2019;22(6):1153-1163.

60. Sharma D, Malik A, Guy CS, Karki R, Vogel P, Kanneganti TD. Pyrin inflammasome regulates tight junction integrity to restrict colitis and tumorigenesis. Gastroenterology. 2018;154(4):948-964.e8.

61. el Marjou F, et al. Tissue-specific and inducible Cre-mediated recombination in the gut epithelium. Genesis. 2004;39(3):186-193.

62. Shen L, Turner JR. Actin depolymerization disrupts tight junctions via caveolae-mediated endocytosis. Mol Biol Cell. 2005;16(9):3919-3936.

63. Shen L, Weber CR, Turner JR. The tight junction protein complex undergoes rapid and continuous molecular remodeling at steady state. J Cell Biol. 2008;181(4):683-695

64. Haljamäe H, Jodal M, Lundgren O. Countercurrent multiplication of sodium in intestinal villi during absorption of sodium chloride. Acta Physiol Scand. 1973;89(4):580-593.

65. Kleinewietfeld M, et al. Sodium chloride drives autoimmune disease by the induction of pathogenic TH17 cells. Nature. 2013;496(7446):518-522.

66. Wu C, et al. Induction of pathogenic TH17 cells by inducible salt-sensing kinase SGK1. Nature. 2013;496(7446):513-517.

67. Hernandez AL, et al. Sodium chloride inhibits the suppressive function of $\mathrm{FOXP3}^{+}$regulatory $\mathrm{T}$ cells. J Clin Invest. 2015;125(11):4212-4222.

68. Aguiar SLF, et al. High-salt diet induces IL-17dependent gut inflammation and exacerbates colitis in mice. Front Immunol. 2017;8:1969.

69. Guo HX, et al. Sodium chloride exacerbates dextran sulfate sodium-induced colitis by tuning proinflammatory and antiinflammatory lamina propria mononuclear cells through p38/ MAPK pathway in mice. World J Gastroenterol. 2018;24(16):1779-1794.

70. Siddiqui-Jain A, et al. CX-4945, an orally bioavailable selective inhibitor of protein kinase CK2, inhibits prosurvival and angiogenic signaling and exhibits antitumor efficacy. Cancer Res. 2010;70(24):10288-10298.

71. Ferguson AD, et al. Structural basis of CX-4945 binding to human protein kinase CK2. FEBS Lett. 2011;585(1):104-110.

72. Pinto D, Robine S, Jaisser F, El Marjou FE, Louvard D. Regulatory sequences of the mouse villin gene that efficiently drive transgenic expression in immature and differentiated epithelial cells of small and large intestines. J Biol Chem. 1999;274(10):6476-6482.

73. France M, Skorich E, Kadrofske M, Swain GM, Galligan JJ. Sex-related differences in small intestinal transit and serotonin dynamics in highfat-diet-induced obesity in mice. Exp Physiol. 2016;101(1):81-99.

74. Nik AM, Carlsson P. Separation of intact intestinal epithelium from mesenchyme. BioTechniques. 2013;55(1):42-44.

75. Clayburgh DR, et al. Epithelial myosin light chain kinase-dependent barrier dysfunction mediates $\mathrm{T}$ cell activation-induced diarrhea in vivo. J Clin Invest. 2005;115(10):2702-2715.

76. Junqueira LC, Bignolas G, Brentani RR. Picrosirius staining plus polarization microscopy, a specific method for collagen detection in tissue sections. Histochem J. 1979;11(4):447-455. 